PACS numbers: 11.10.Cd, 10.30.Ly and 11.10.Gh

\title{
Noether Charges for Self-interacting Quantum Field Theories in Curved Spacetimes with a Killing-vector
}

\author{
Stefan Hollands* \\ Enrico Fermi Institute, Univ. of Chicago, 5640 Ellis Avenue, \\ Chicago, IL 60637 - 1433, USA
}

October 28, 2018

\begin{abstract}
We consider a self-interacting, perturbative Klein-Gordon quantum field in a curved spacetime admitting a Killing vector field. We show that the action of this spacetime symmetry on interacting field operators can be implemented by a Noether charge which arises, in a certain sense, as a surface integral over the time-component of some interacting Noether current-density associated with the Killing field. The proof of this involves the demonstration of a corresponding set of Ward identities. Our work is based on the perturbative construction by Brunetti and Fredenhagen (Commun.Math.Phys. 208 (2000) 623-661) of self-interacting quantum field theories in general globally hyperbolic spacetimes.
\end{abstract}

KEYWORDS: Quantum field theory, perturbation theory, Noether charges, Killing vectors

*Electronic mail: stefan@bert.uchicago.edu 


\section{Intorduction}

It is widely believed that the theory of quantum fields in a classical curved spacetime background (for an introduction to this subject, see e.g. [18, 12]) provides an excellent description of physical processes in which both gravitational effects as well as the quantum nature of matter on the microscopic level are important, but in which quantum effects of the gravitational field and back-reaction are not important. Among others, it led to the spectacular prediction [8], by Hawking in 1975, that black holes "are not black", but instead emit thermal radiation at temperature $T_{H}=\frac{\kappa}{2 \pi}$, where $\kappa$ is the surface gravity of the horizon. Moreover, the analysis of the conceptual basis of quantum field theory (QFT) in curved spacetime has also lead to a reassessment of the general structure of QFT, since it forces one to emphasize the principle of locality, and abandon concepts such as preferred global vacuum states, the conventional notion of "particle", unitary time-evolution, etc.

Most effects predicted by QFT in curved spacetime can already be seen in linear (i.e., non self-interacting) models, and in fact the vast majority of the work done in the subject has been devoted to such models. However, they are not realistic and one therefore also ought to consider self-interacting quantum fields on curved backgrounds (of course one expects that the essential physical effects predicted by linear models remain). Unfortunately, it does not seem to be clear at present - even in Minkowski space - just what a self-interacting quantum field really is, phrased in sensible mathematical terms, exept in some rather special models in low spacetime dimensions. On the other hand, it has long been known, at least in Minkowski space, how to construct self-interacting quantum field theory models on the level of perturbation theory. The challenge to construct perturbatively also self-interacting quantum fields in curved spacetime has been taken up by several authors [4, 5, 3, most recently ${ }^{1}$ by [3, who have developed a variant of the causal approach to perturbation theory [7, 17, 1, 15] for curved spacetimes, thereby arriving at the same classification of theories into (perturbatively) renormalizable and non-renormalizable ones as in Minkowski space. ${ }^{2}$

One interesting result of [3] (which seems to be new even in flat spacetime) is that it is always possible construct algebras $\mathfrak{A}_{\mathscr{L}}(\mathcal{O})$ of interacting quantum fields (given by

\footnotetext{
${ }^{1}$ We note that, while [3] applies to any smooth, globally hyperbolic spacetime, this is not the case for the construction by [4, 5], who approach the problem from the point of view of Euclidean spaces rather than Lorentzian spacetimes. Now, if a Lorentzian spacetime can be viewed as a real section of some complex space which also posses a real Euclidean section, then the quantities of interest for the interacting theory in that Lorentzian spacetime can be obtained, via analytic continuation, from suitably defined corresponding quantities in the Euclidean section. However, apart from the static ones, there are essentially no curved spacetimes with this property and we do not want to restrict ourselves to such a limited class of spacetimes here.

${ }^{2}$ We must point out that the finite renormalization ambiguities found in [3], even for renormalizable theories, are much greater than for the corresponding theory in Minkowski space: Instead of free parameters they consist of free functions. This problem has been resolved in [10], but for the purposes of this article we can ignore this issue.
} 
formal power series in the coupling strength) localized in some bounded region $\mathcal{O}$ in spacetime, where the subscript $\mathscr{L}$ indicates that the theory is defined by a local interaction Lagrangian $\mathscr{L}$ (the construction of these algebras will be reviewed in some detail in Sec. 2). These local algebras have the property that $\mathfrak{A}_{\mathscr{L}}\left(\mathcal{O}_{1}\right) \subset \mathfrak{A}_{\mathscr{L}}\left(\mathcal{O}_{2}\right)$ if $\mathcal{O}_{1} \subset \mathcal{O}_{2}$, and one can therefore define the algebra of all observables of the theory as the inductive limit $\mathfrak{A}_{\mathscr{L}}=\cup_{\mathcal{O}} \mathfrak{A}_{\mathscr{L}}(\mathcal{O})$. This is quite remarkable, because it means that one can perturbatively construct the theory on the level of observables even in cases where an $S$-matrix does not properly exist because of incurable infrared divergences.

In this work we want to study a self interacting Klein-Gordon field on globally hyperbolic spacetimes $\left(M, \mathbf{g}_{a b}\right)$ which have an everywhere non-vanishing Killing vectorfield $\xi^{a}$ and for which there exists a $\xi^{a}$-invariant quasifree Hadamard state (an explanation of this term will be given below) for the corresponding linear quantum field ${ }^{3}$. This class of spacetimes includes many examples of physical interest, for example the exterior of the Schwarzschild solution to Einstein's equation. Under these conditions, there exists an action of the corresponding 1-parameter symmetry group of $M$ by automorphsims $\alpha \mathscr{L}, t$ on the algebra of interacting fields $\mathfrak{A}_{\mathscr{L}}$, such that $\alpha_{\mathscr{L}, t}\left(\mathfrak{A}_{\mathscr{L}}(\mathcal{O})\right)=\mathfrak{A}_{\mathscr{L}}\left(\psi^{t} \mathcal{O}\right)$, where $\psi^{t}$ is the flow of $\xi^{a}$.

Now, it is a well-established feature of QFT in Minkowski spacetime that the (infinitesimal) action of a 1-parameter family of (unbroken) symmetries of a theory can be implemented, at least locally, by a charge operator which arises as a surface integral of a corresponding conserved Noether current-density. One expects that this should also hold in the present situation, where the 1-parameter group of symmetries in question now arises from the Killing vector field of the underlying curved spacetime. The aim of this paper is to show that this expectation is indeed correct. Namely, we show that for any bounded region $\mathcal{O}$ and any globally hyperbolic region $\widehat{\mathcal{O}}$ "strongly containing" $\mathcal{O}$, there is an operator $Q_{\mathscr{L}} \in \mathfrak{A}_{\mathscr{L}}(\widehat{\mathcal{O}})$ such that the infinitesimal action of $\alpha_{\mathscr{L}, t}$ on any element $a \in \mathfrak{A}_{\mathscr{L}}(\mathcal{O})$ is given by $\left[Q_{\mathscr{L}}, a\right]$ (we mean the commutator). Moreover, $Q_{\mathscr{L}}$ arises, in a certain sense, as a surface integral of the time-component of a covariantly conserved interacting Noether current-density $J_{\mathscr{L}}^{a}$ over an arbitrarily chosen Cauchy surface $\widehat{\Sigma}$ of $\widehat{\mathcal{O}}$. (We say that $\widehat{\mathcal{O}}$ strongly contains $\mathcal{O}$ if there is a Cauchy-surface $\widehat{\Sigma}$ of $\widehat{\mathcal{O}}$ and an open set $U \subset \widehat{\Sigma}$ such that the closure of $J(\mathcal{O}) \cap \widehat{\Sigma}$ is contained in $U$.)

The main part of our proof of this result consists in showing that the perturbatively defined interacting current $J_{\mathscr{L}}^{a}$ is covariantly conserved, which in turn is equivalent to the validity of a corresponding set of Ward identities between certain time ordered products. A general framework to prove Ward identities within the causal approach was developed by Dütsch and Fredenhagen [6] (they treated the case of the global $U(1)$-currents for QED

\footnotetext{
${ }^{3}$ We remark that, since all our constructions are local, all the results in this paper still hold under the weaker assumption that there exists a $\xi^{a}$-invariant Hadamard state for every globally hyperbolic set $\mathcal{O}$ in $M$ with compact closure. Hence the assumption that there is a globally defined $\xi^{a}$-invariant quasifree Hadamard state (which might not exist, due to infrared problems) is not essential.
} 
in Minkowski spacetime), we here adopt their strategy. The crucial step in the proof is to remove the anomaly. In the case studied by [6] (and similarly [13, 9], where the Ward identities for the interacting stress tensor in Minkowski space are treated), this is done by an argument based on momentum space techniques. These are not available in the case studied here, and one therefore has to proceed by an entirely different argument.

We remark that, while we explitely deal only with a scalar self-interacting field, a generalization of our results to other types of fields should be straightforward.

Our plan for this paper is as follows: In Sec. 2 we review the quantization of a linear Klein-Gordon field on a curved spacetime. After that, we recall the notion of Wick products in curved space and then explain in some detail the construction of interacting fields, the algebras $\mathfrak{A}_{\mathscr{L}}(\mathcal{O})$, etc. in curved spacetime (here we follow [3]). In Sec. 3, we define the automorphic action $\alpha_{\mathscr{L}, t}$ of the group of spacetime symmetries on the observables in the interacting theory. We then show that this action is implemented by a local charge operator $Q_{\mathscr{L}}$. The Ward identities for $J_{\mathscr{L}}^{a}$ are proved in the Appendix.

Notations and conventions: $\left(M, \mathbf{g}_{a b}\right)$ denotes a globally hyperbolic, time-oriented four dimensional curved spacetime of signature $+2 . J^{ \pm}(\mathcal{O})$ is the causal future respectively past of a subset $\mathcal{O} \subset M$, and $J(\mathcal{O})=J^{+}(\mathcal{O}) \cup J^{-}(\mathcal{O})$. By $D(\mathcal{O})$ we mean the domain of dependence of a region $\mathcal{O} \subset M$, defined as the set of all points $x$ such that every future or every past directed inextentible causal curve starting at $x$ intersects $\mathcal{O}$. The metric volume element is denoted by $\mu_{\mathbf{g}}$ and the wave operator in curved spacetime by $\square_{\mathbf{g}}=\mathbf{g}^{a b} \nabla_{a} \nabla_{b}$. $\mathscr{D}(M)$ denotes the space of compactly supported (complex-valued) testfunctions on $M$ and $\mathscr{D}^{\prime}(M)$ is the corresponding dual space of distributions. We shall also find it useful to use a multi-index notation. If $\alpha=\left(\alpha_{1}, \ldots, \alpha_{n}\right)$ is a multi-index, then we set $\alpha !=\prod_{i} \alpha_{i}$ !.

\section{Review of QFT in CST}

\subsection{Quantization of linear scalar fields}

The theory of a free quantized Klein-Gordon field in curved spacetime can be formulated in various ways. For our purposes, it is essential to formulate the theory within the so-called "algebraic approach" (see, for example [18, 12]). In this approach, one starts from an abstract *-algebra $\mathfrak{A}$ (with unit), which is generated by certain expressions in the smeared quantum field, $\Phi(f)$, where $f$ is a test function. In [18, 12, expressions of the form $e^{i \Phi(f)}$ were considered. The main advantage of working with such expressions is that the so-obtained algebra then has a norm (in technical terms, it is a $C^{*}$-algebra). Defining the algebra $\mathfrak{A}$ in that way would however be inconvenient for our purposes. Instead, we shall take $\mathfrak{A}$ to be the *-algebra generated by the identity and the smeared field operators $\Phi(f)$ themselves, subject to the following relations:

Linearity: The map $f \mapsto \Phi(f)$ is complex linear.

Klein-Gordon Equation: $\Phi\left(\left(\square_{\mathbf{g}}-m^{2}\right) f\right)=0$ for all $f \in \mathscr{D}(M)$. 
Hermiticity: $\Phi(f)^{*}=\Phi(\bar{f})$ for all $f \in \mathscr{D}(M)$.

Commutation relations: $\left[\Phi\left(f_{1}\right), \Phi\left(f_{2}\right)\right]=i \Delta\left(f_{1}, f_{2}\right) \mathbb{1}$ for all $f_{1}, f_{2} \in \mathscr{D}(M)$, where $\Delta$ is the causal propagator (commutator function), defined as the difference $\Delta_{A}-\Delta_{R}$ between the uniquely determined advanced and retarded fundamental solutions of the Klein-Gordon operator $\square_{\mathrm{g}}-m^{2}$.

The so-obtained algebra $\mathfrak{A}(M, \mathbf{g})$ is now no longer a $C^{*}$-algebra, because of the unbounded nature of the smeared quantum fields $\varphi(f)$. This will however not be relevant in the following. We will often use the informal notation

$$
\Phi(f)=\int_{M} \Phi(x) f(x) \mu_{\mathbf{g}}(x),
$$

for the smeared quantum fields, and also for other distributions. The local algebras $\mathfrak{A}(\mathcal{O})$ are by definition the subalgebras of $\mathfrak{A}$ generated by quantum fields smeared with testfunctions supported in $\mathcal{O} \subset M$. Clearly these local algebras fulfill isotony, $\mathfrak{A}\left(\mathcal{O}_{1}\right) \subset$ $\mathfrak{A}\left(\mathcal{O}_{2}\right)$ if $\mathcal{O}_{1} \subset \mathcal{O}_{2}$. Since $\Delta\left(x_{1}, x_{2}\right)=0$ for spacelike related points, the local algebras also satisfy spacelike commutativity, $\left[\mathfrak{A}\left(\mathcal{O}_{1}\right), \mathfrak{A}\left(\mathcal{O}_{2}\right)\right]=\{0\}$ if $\mathcal{O}_{1}$ and $\mathcal{O}_{2}$ are spacelike.

A state in the algebraic framework is by definition a positive, normalized linear functional on $\omega: \mathfrak{A} \rightarrow \mathbb{C}$, i.e., a linear functional with the properties $\omega\left(a^{*} a\right) \geq 0$ for all $a \in \mathfrak{A}$ and $\omega(\mathbb{1})=1$. The algebraic notion of state is related to the usual Hilbert-space notion of state via the so-called "GNS-theorem", which says that for any algebraic state $\omega$, there exists a representation $\pi_{\omega}$ of $\mathfrak{A}$ on a Hilbert space $\mathscr{H}_{\omega}$, and a cyclic vector $\left|\Omega_{\omega}\right\rangle$ such that $\omega(a)=\left\langle\Omega_{\omega} \mid \pi_{\omega}(a) \Omega_{\omega}\right\rangle$ for all $a \in \mathfrak{A}$. If the state in question had been obtained from some set $u_{\lambda}$ of positive frequency solutions to the Klein-Gordon equation, then the GNSconstruction gives the usual representation of the field operators on Fock space (with $\left|\Omega_{\omega}\right\rangle$ the Fock vacuum), $\Phi(x)=\sum_{\lambda} a_{\lambda} u_{\lambda}(x)+a_{\lambda}^{+} u_{\lambda}^{+}(x)$, where $a_{\lambda}^{+}$are the creation operators corresponding to the modes $u_{\lambda}$. The multilinear functionals on $\mathscr{D}(M)$ defined by

$$
\omega^{(n)}\left(f_{1}, \ldots, f_{n}\right)=\omega\left(\Phi\left(f_{1}\right) \ldots \Phi\left(f_{n}\right)\right)
$$

are called "n-point functions" (of the state $\omega$ ). A state is called "quasifree" if it has a vanishing one-point function and vanishing truncated $n$-point functions for $n>2$. Prime examples for quasifree states are the states obtained from some set of positive frequency solutions $u_{\lambda}$. A state is called "globally Hadamard" if its two-point function has no spacelike singularities and if its symmetrized two-point function locally has the form of a Hadamard fundamental solution, $H$, given by

$$
H\left(x_{1}, x_{2}\right)=u\left(x_{1}, x_{2}\right) \mathrm{P}\left(\sigma^{-1}\right)+v\left(x_{1}, x_{2}\right) \ln |\sigma|+w\left(x_{1}, x_{2}\right) .
$$

Here, $\sigma$ is the signed, squared geodesic distance between $x_{1}$ and $x_{2}, u$ and $v$ are certain smooth, symmetric functions constructed from the metric, "P" means the principal value, and $w$ is a smooth, symmetric function depending on the state. For a mathematically 
precise definition of these quantities and of the statement that "there are no spacelike singularities", we refer to [12. There exists an alternative, equivalent characterization of globally Hadamard states in terms of the wave front set (for a definition of this concept, see [11]) of their two-point function, due to Radzikowski [14, which plays a crucial rôle in the construction of normal orderd Wick products 2] (given in the next subsection), and of time ordered products [3] in curved spacetime. Namely, a state is Hadamard, if its two-point function has the following wave front set:

$$
\mathrm{WF}\left(\omega^{(2)}\right)=\left\{\left(x_{1}, k_{1}, x_{2},-k_{2}\right) \in\left(T^{*} M\right)^{2} \backslash\{\mathbf{0}\} \mid\left(x_{1}, k_{1}\right) \sim\left(x_{2}, k_{2}\right), k_{2} \triangleright 0\right\} .
$$

Here, the notation $\left(x_{1}, k_{1}\right) \sim\left(x_{2}, k_{2}\right)$ means that $x_{1}$ and $x_{2}$ can be joined by a null geodesic and that $k_{1}$ and $k_{2}$ are cotangent and coparallel to this geodesic. $k \triangleright 0$ means that $k_{a}$ is future pointing.

\subsection{Review of Wick products of free fields in curved spacetime}

Brunetti, Fredenhagen and Köhler [2] gave a construction of Wick products of free fields in curved spacetime (with respect to some arbitrarily chosen quasi-free reference Hadamard state $\omega)$, which generalizes the well-known construction of Wick products in Minkowski space. These quantities are not already contained in the algebra $\mathfrak{A}$ of free fields and form the starting point for the perturbative construction of the interacting fields, which will be reviewed in the next subsection.

To begin with, the authors of [2] first define "point-split Wick products" by

$$
: \Phi\left(x_{1}\right) \ldots \Phi\left(x_{n}\right): \omega=\left.\frac{\delta^{n}}{i^{n} \delta f\left(x_{1}\right) \ldots \delta f\left(x_{n}\right)} \exp \left[\frac{1}{2} \omega^{(2)}(f, f) \mathbb{1}+i \Phi(f)\right]\right|_{f=0},
$$

where the field operators $\Phi$ on the right side of this equation mean the representers of the corresponding algebraic elements in the GNS-representation of $\omega$. (We note here that this normal ordering prescription is equivalent to usual one defined in terms of creation and destruction operators. More precisely, suppose that the state $\omega$ in question had been obtained from some set $u_{\lambda}$ of positive frequency solutions to the Klein-Gordon equation. One could then normal order the product $\Phi\left(x_{1}\right) \ldots \Phi\left(x_{n}\right)$ by shifting all the creation operators $a_{\lambda}^{+}$(corresponding to the modes $u_{\lambda}$ ) in that expression the left of all destruction operators. It is not hard to see that the operator obtained in this way is identical to the operator : $\Phi\left(x_{1}\right) \ldots \Phi\left(x_{n}\right):_{\omega}$ defined above.) [2] demonstrated that the above Wick products are well-defined operator-valued distributions on $M^{n}=M \times \cdots \times M$ ( $n$ factors) on a dense invariant domain $D_{\omega}$ in the GNS-Hilbert space $\mathscr{H}_{\omega}$, and that they posses well-defined restrictions to all partial diagonals in $M^{n}$. (A "partial diagonal" is a subset of $M^{n}$ of the form

$$
\left.\Delta_{n_{1}, \ldots, n_{j}}(M)=\left\{(\underbrace{x_{1}, \ldots, x_{1}}_{n_{1} \text { times }}, \ldots, \underbrace{x_{j}, \ldots, x_{j}}_{n_{j} \text { times }}) \mid x_{i} \in M, i=1, \ldots, j\right\} \cong M^{j} .\right)
$$


The restriction of the point-split Wick product to such a partial diagonal is called a "multi-local Wick product" and is denoted by : $\Phi^{n_{1}}\left(x_{1}\right) \ldots \Phi^{n_{j}}\left(x_{j}\right):_{\omega}$. The restriction to the total diagonal, $\Delta_{n}(M)$, of the point-split Wick product is simply called a "Wick monomial" and is denoted by : $\Phi^{n}(x):_{\omega}$. We remark that while it is possible to define the point-split Wick products for any quasifree state, this is not so for the above Wick monomials (resp. multi-local Wick monomials). For the latter, it is crucial that one uses Hadamard states.

We end this subsection by introducing some quantities and notions related to the above normal ordered Wick products. A linear combination of Wick monomials with coefficients in $\mathscr{D}(M)$ is called a "Wick polynomial". Wick monomials and Wick polynomials involving derivatives can be defined in a similar fashion from point-split Wick products of differentiated free fields. For any Wick-polynomial $A$ without derivatives, we define a Wick polynomial $\frac{\partial A}{\partial \Phi}$ by

$$
i \frac{\partial A}{\partial \Phi}(x) \Delta(x, y)=[A(x), \Phi(y)]
$$

For later purposes, we also find it convenient to define the $\operatorname{degree,~} \operatorname{deg}(A)$, of a Wick monomial $A$ as the number of free field factors plus the number of derivatives in $A$.

\subsection{Review of perturbative interacting quantum field theory in curved spacetime}

The building blocks of perturbative interacting QFT (in the real-time formalism) are the time ordered products of Wick polynomials (in curved spacetime, the latter are defined with respect to some arbitrary reference Hadamard state $\omega$, which shall be kept fixed for the rest of this section). The time ordered products are formally defined by

$$
\text { “T }\left(A_{1}\left(x_{1}\right) \ldots A_{n}\left(x_{n}\right)\right)=\sum_{\operatorname{perm} \pi} \vartheta\left(x_{\pi(1)}^{0}-x_{\pi(2)}^{0}\right) \ldots \vartheta\left(x_{\pi(n-1)}^{0}-x_{\pi(n)}^{0}\right) A_{1}\left(x_{\pi(1)}\right) \ldots A_{n}\left(x_{\pi(n)}\right) ",
$$

where $x^{0}$ is a global time-coordinate on $M, \vartheta$ is the distribution defined by

$$
\vartheta(t)=\left\{\begin{array}{l}
1 \text { if } t \geq 0 \\
0 \text { otherwise }
\end{array}\right.
$$

and $A_{i}, i=1, \ldots, n$ are Wick polynomials. Formula (11) for the time ordered products cannot in general be taken at face value, since the operator products appearing on the right hand side of this equation are in general too (short-distance-) singular to be multiplied by $\vartheta$-distributions, and therefore this equation is meaningless from a mathematical point of view. ${ }^{4}$ There are however ways to construct mathematically well-defined, "renormalized"

\footnotetext{
"One aspect of this is that, if one naively 'calculates' the above formal expression for the time ordered products (e.g. by applying what is usually called the "naive Feynman rules") then one is faced with ultraviolet divergences which lead to meaningless infinite results.
} 
operators $\mathscr{T}\left(A_{1}\left(x_{1}\right) \ldots A_{n}\left(x_{n}\right)\right)$ which have the essential features of 'time orderedness' formally shared by the ill-defined expression on the right hand side of Eq. (11). The process of constructing such time ordered products as well-defined operators on $\mathscr{H}_{\omega}$ (with some common, dense and invariant domain) is usually referred to as "renormalization". This will be addressed in some more detail below; for the moment we assume that welldefined time ordered products have been constructed, and proceed by explaining how one can locally define interacting quantum fields, algebras of observables etc. from these (renormalized) time ordered products.

The interaction of a Klein-Gordon field with itself is described by an interaction Lagrangian $\mathscr{L}(x)=\lambda A(x)$, where $\lambda$ is the coupling strength and $A$ is a Wick polynomial. In order to get a renormalizable theory $A$ must be a Wick polynomial of degree less or equal than four. ${ }^{5}$ The corresponding $S$-matrix, $S[\mathscr{L}]$, is defined, again formally, in terms of the renormalized time ordered products by the so-called "Gell Mann-Low formula" (viewed as a formal power series in the coupling strength $\lambda$ ),

$$
" S[\mathscr{L}]=\mathbb{1}+\sum_{n=1}^{\infty} \frac{i^{n}}{n !} \int \mathscr{T}\left(\mathscr{L}\left(x_{1}\right) \ldots \mathscr{L}\left(x_{n}\right)\right) \mu_{\mathbf{g}}\left(x_{1}\right) \ldots \mu_{\mathbf{g}}\left(x_{n}\right) "
$$

In Minkowski space, one obtains from the $S$-matrix-provided it exists (i.e., there is a suitable sense in which the above integrals converge at arbitrary order in perturbation theory) - the scattering amplitudes between asymptotic incoming and outgoing states. There are however (otherwise perfectly reasonable) theories, for which the $S$-matrix does not properly exist, due to uncurable infrared divergences. Moreover, in curved spacetimes which are not asymptotically flat in the remote past and future (for example, spacetimes with an initial big-bang singularity), there is no well defined notion of a scattering process to begin with, regardless as to whether the above expression for the $S$-matrix makes good mathematical sense or not. Although we think that for this reason the $S$-matrix does not occupy any fundamental status in a generic curved spacetime, it is still of some value as a technical tool. For example, it can be used to construct the interacting theory locally on the algebraic level, as we shall briefly recall now (see ref. [3] for details).

For this purpose, one first introduces a local version of the $S$-matrix which is defined by the same formula as above, Eq. (2), but with the interaction Lagrangian replaced by the local quantity $g(x) \mathscr{L}(x)$ where $g$ is a smooth function of compact support. As a consequence the volume integrals in the expression for this local $S$-matrix now trivially converge. For any Wick-polynomial $A(x)$ of free field operators one then introduces a

\footnotetext{
${ }^{5}$ In order for the interaction to be local, one must further require that $\mathscr{L}(x)$ should be 'locally defined in terms of the metric' (for a precise mathematical formulation of this requirement see e.g. [18] [item 2) on p. 89]). Such a requirement further reduces the possible form of $\mathscr{L}(x)$. Further restrictions come from dimensional considerations.
} 
corresponding interacting field operator (thought of as formal power series in $\lambda$ ) by

$$
A_{g} \mathscr{L}(f)=\left.S[g \mathscr{L}]^{-1} \frac{\partial}{i \partial t} S[g \mathscr{L}+t f A]\right|_{f=0}, \quad f \in \mathscr{D}(M) .
$$

It is possible to expand the interacting field operators in terms of so-called "totally retarded products" (or $\mathscr{R}$-products):

$$
A_{g \mathscr{L}}(y)=\sum_{n=0}^{\infty} \frac{i^{n}}{n !} \int \mathscr{R}\left(A(y) ; \mathscr{L}\left(x_{1}\right) \ldots \mathscr{L}\left(x_{n}\right)\right) g\left(x_{1}\right) \ldots g\left(x_{n}\right) \mu_{\mathbf{g}}\left(x_{1}\right) \ldots \mu_{\mathbf{g}}\left(x_{n}\right),
$$

where

$$
\begin{aligned}
\mathscr{R}\left(A(y) ; A_{1}\left(x_{1}\right) \ldots A_{n}\left(x_{n}\right)\right)= & \\
& \sum_{I \subset\{1, \ldots, n\}}(-1)^{|I|} \mathscr{T}\left(\prod_{i \in I} A_{i}\left(x_{i}\right)\right)^{+} \mathscr{T}\left(A(y) \prod_{j \in I^{c}} A_{j}\left(x_{j}\right)\right) .
\end{aligned}
$$

The $\mathscr{R}$-products are symmetric in $x_{1}, \ldots, x_{n}$ and have support in the set $\left\{\left(y, x_{1}, \ldots, x_{n}\right) \mid\right.$ $\left.x_{j} \in J^{-}(y), j=1, \ldots, n\right\}$. The commutator of two interacting fields is given by

$$
\begin{aligned}
& {\left[A_{1 g \mathscr{L}}\left(y_{1}\right), A_{2 g} \mathscr{L}\left(y_{2}\right)\right]=\sum_{n=0}^{\infty} \frac{i^{n}}{n !} \int \mu_{\mathbf{g}}\left(x_{1}\right) \ldots \mu_{\mathbf{g}}\left(x_{n}\right) g\left(x_{1}\right) \ldots g\left(x_{n}\right) \times} \\
& \left(\mathscr{R}\left(A_{1}\left(y_{1}\right) ; A_{2}\left(y_{2}\right) \mathscr{L}\left(x_{1}\right) \ldots \mathscr{L}\left(x_{n}\right)\right)-\mathscr{R}\left(A_{2}\left(y_{2}\right) ; A_{1}\left(y_{1}\right) \mathscr{L}\left(x_{1}\right) \ldots \mathscr{L}\left(x_{n}\right)\right)\right),
\end{aligned}
$$

see [6] for a proof of this formula.

We come to the definition of local algebras $\mathfrak{A}_{\mathscr{L}}(\mathcal{O})$ of observables in the interacting theory [3, 6]. Let $\mathcal{O}$ be a bounded open set in $M$ with compact closure. For any compactly supported, smooth function $g$ on $M$ such that $g \uparrow \mathcal{O}=1$ (we denote the set of such functions by $\vartheta(\mathcal{O})$ ) one first introduces the algebra

$$
\left.\mathfrak{A}_{g \mathscr{L}}(\mathcal{O})=\left\langle A_{g \mathscr{L}}(f)\right| f \in \mathscr{D}(\mathcal{O}), \quad A \text { a Wick-polynomial }\right\rangle
$$

Now one can prove that if $g^{\prime}$ is another function in $\vartheta(\mathcal{O})$, then there exists a unitary $V$ such that

$$
A_{g \mathscr{L}}(f) V=V A_{g^{\prime} \mathscr{L}}(f)
$$

for all $f \in \mathscr{D}(\mathcal{O})$ and any Wick polynomial $A$, thus showing that the algebraic structure of $\mathfrak{A}_{g \mathscr{L}}(\mathcal{O})$ is actually independent of the choice of $g$. This observation leads one to the following definition for the algebras of observables $\mathfrak{A}_{\mathscr{L}}(\mathcal{O})$ : Let $\mathscr{V}_{g, g^{\prime}}(\mathcal{O})$ be the set of 
unitaries satisfying Eq. (7) for all $f \in \mathscr{D}(\mathcal{O})$ and all Wick polynomials $A$. Then the local algebras $\mathfrak{A}_{\mathscr{L}}(\mathcal{O})$ are by definition the algebras generated by the set of all families

$$
a=\left(a_{g}\right)_{g \in \vartheta(\mathcal{O})}, \quad a_{g} \in \mathfrak{A}_{g \mathscr{L}}(\mathcal{O})
$$

with the property that $a_{g} V=V a_{g^{\prime}}$ for all $V \in \mathscr{V}_{g, g^{\prime}}(\mathcal{O})$ and for any two functions $g, g^{\prime}$ in $\vartheta(\mathcal{O})$. The product and ${ }^{*}$-operation in this algebra are the obvious ones:

$$
(a b)_{g}:=a_{g} b_{g}, \quad\left(a^{*}\right)_{g}:=a_{g}^{*} .
$$

By definition, the algebra $\mathfrak{A}_{\mathscr{L}}(\mathcal{O})$ contains for example the elements defined by $a=$ $\left(A_{g \mathscr{L}}(f)\right)_{g \in \vartheta(\mathcal{O})}$, where $f \in \mathscr{D}(\mathcal{O})$ and where $A$ is an arbitrary Wick polynomial. In the following we denote such elements by $A_{\mathscr{L}}(f)$. It is clear that if $\mathcal{O}_{1} \subset \mathcal{O}_{2}$ are two spacetime regions with compact closure, then every element in $\mathfrak{A}_{\mathscr{L}}\left(\mathcal{O}_{1}\right)$ can naturally be identified with an element in $\mathfrak{A}_{\mathscr{L}}\left(\mathcal{O}_{2}\right)$, thus $\mathfrak{A}_{\mathscr{L}}\left(\mathcal{O}_{1}\right) \subset \mathfrak{A}_{\mathscr{L}}\left(\mathcal{O}_{2}\right)$. One may therefore define the algebra of all observables in the interacting theory as the inductive limit, $\mathfrak{A}_{\mathscr{L}}=\cup_{\mathcal{O}} \mathfrak{A}_{\mathscr{L}}(\mathcal{O})$. It follows from Eq. (6) and the support properties of the $\mathscr{R}$-products that

$$
\left[A_{1 \mathscr{L}}\left(f_{1}\right), A_{2 \mathscr{L}}\left(f_{2}\right)\right]=0
$$

if the support of $f_{1}$ is spacelike related to the support of $f_{2}$. This means that the net $\mathfrak{A}_{\mathscr{L}}(\mathcal{O})$ satisfies spacelike commutativity.

In the previous paragraphs, we have indicated how quantities of interest in the interacting theory can be constructed from the time ordered products. In the remaining part of this subsection we briefly address the issue of constructing the time ordered products themselves. Our discussion follows [3], who have generalized the methods of causal perturbation theory [7, 1, 17, 15, 16] in Minkowski space to general globally hyperbolic curved spacetimes. The main idea behind the causal approach is to construct the time ordered products by an inductive process based on their causal factorization properties. The induction proceeds by the order $n$ in perturbation theory (= number of factors in the time ordered products). At first order, one sets $\mathscr{T}(A(x)):=A(x)$ for any Wick monomial $A$. The inductive assumptions on the time ordered products at a given order $n \geq 1$ (they are satisfied at first order) are the following:

Well-definedness: The time ordered products are well-defined operator valued distributions on some dense, invariant domain $D_{\omega} \subset \mathscr{H}_{\omega}$.

\section{Causal factorization:}

$$
\mathscr{T}\left(A_{1}\left(x_{1}\right) \ldots A_{n}\left(x_{n}\right)\right)=\mathscr{T}\left(A_{1}\left(x_{1}\right) \ldots A_{n}\left(x_{k}\right)\right) \mathscr{T}\left(A_{1}\left(x_{k+1}\right) \ldots A_{n}\left(x_{n}\right)\right)
$$

if

$$
x_{j} \notin J^{-}\left(x_{i}\right), \quad \text { for all } i=1, \ldots, k \text { and } j=k+1, \ldots, n \text {, }
$$


i.e., the time ordered products factorize if every point in one set of points is in the past of another set of points.

Permutation symmetry: The time ordered product are symmetric under permutations of the arguments.

\section{Unitarity:}

$$
\mathscr{T}\left(A_{1}\left(x_{1}\right) \ldots A_{n}\left(x_{n}\right)\right)^{+}=\sum_{\mathcal{P}}(-1)^{n+|\mathcal{P}|} \prod_{I \in \mathcal{P}} \mathscr{T}\left(\prod_{i \in I} A_{i}\left(x_{i}\right)^{+}\right),
$$

where $\mathcal{P}$ runs over all partitions of $\{1, \ldots, n\}$ into pairwise disjoint subsets.

Remark: This condition is equivalent to requiring that the $S$-matrix is unitary in the sense of formal power series of operators.

Wick expansion: Let $A_{i}, i=1, \ldots, n$ be Wick polynomials without derivatives. Then

$$
\mathscr{T}\left(A_{1}\left(x_{1}\right) \ldots A_{n}\left(x_{n}\right)\right)=\sum_{\alpha} t_{n}^{\alpha}\left(x_{1}, \ldots, x_{n}\right) / \alpha !: \Phi^{\alpha_{1}}\left(x_{1}\right) \ldots \Phi^{\alpha_{n}}\left(x_{n}\right):_{\omega},
$$

where

$$
t_{n}^{\alpha}\left(x_{1}, \ldots, x_{n}\right)=\left\langle\Omega_{\omega} \mid \mathscr{T}\left(\frac{\partial^{\alpha_{1}} A_{1}}{\partial \Phi^{\alpha_{1}}}\left(x_{1}\right) \ldots \frac{\partial^{\alpha_{n}} A_{n}}{\partial \Phi^{\alpha_{n}}}\left(x_{n}\right)\right) \Omega_{\omega}\right\rangle .
$$

(That the products between the various distributions in Eq. (9) indeed exist can be derived from the upper bound on the wave front set of the $t_{n}^{\alpha}$, given below, and a similar bound on the wave front set of the Wick products. For details see [3, Microlocal Thm. 0].) In our subsequent discussions, we will often use the following consequence of Eq. (9):

$$
\left[\mathscr{T}\left(A_{1}\left(x_{1}\right) \ldots A_{n}\left(x_{n}\right)\right), \Phi(y)\right]=\sum_{j=1}^{n} \mathscr{T}\left(A_{1}\left(x_{1}\right) \ldots\left[A_{j}\left(x_{j}\right), \Phi(y)\right] \ldots A_{n}\left(x_{n}\right)\right) .
$$

Here, the time ordered products involving commutators on the r.h.s. are understood to mean the expression obtained by replacing $\left[A_{j}\left(x_{j}\right), \Phi(y)\right]$ with $i \Delta\left(x_{j}, y\right) \frac{\partial A_{j}}{\partial \Phi}\left(x_{j}\right)$ and by pulling the commutator functions out of the time ordered product.

Remark: An expansion formula similar to Eq. (9) holds also for time ordered products of Wick polynomials containing differentiated field operators, it can e.g. be found in [13. As for the case of undifferentiated Wick polynomials, this implies Eq. (N3), where it is now understood that the expressions $\left[A_{j}\left(x_{j}\right), \Phi(y)\right]$ are to be written in terms of suitably differentiated commutator functions and suitable sub-Wick polynomials of $A_{j}$, see e.g. [6. 13 .

Microlocal spectrum condition: The wave front set of the scalar time ordered distributions $t_{n}$ defined by

$$
t_{n}\left(x_{1}, \ldots, x_{n}\right)=\left\langle\Omega_{\omega} \mid \mathscr{T}\left(A_{1}\left(x_{1}\right) \ldots A_{n}\left(x_{n}\right)\right) \Omega_{\omega}\right\rangle
$$


is contained in a certain set $\Gamma_{n}^{\mathscr{T}} \subset\left(T^{*} M\right)^{n} \backslash\{\mathbf{0}\}$, which is defined as follows: By definition, a point $\left(x_{1}, k_{1} ; \ldots ; x_{n}, k_{n}\right) \in\left(T^{*} M\right)^{n} \backslash\{\mathbf{0}\}$ is in $\Gamma_{n}^{\mathscr{T}}$ if and only if (a) there exist nullgeodesics $\gamma_{1}, \ldots, \gamma_{m}$ which connect any point $x_{j}$ in the set $\left\{x_{1}, \ldots, x_{n}\right\}$ to some other point in that set, (b) there exists coparallel, cotangent covectorfields $p_{1}, \ldots, p_{m}$ along these geodesics such that $p_{l} \triangleright 0$ if the starting point of $\gamma_{l}$ is not in the causal past of the end point of $\gamma_{l}$, (c) for the covector $k_{j}$ over the point $x_{j}$ it holds that $k_{j}=\sum_{e} p_{e}\left(x_{j}\right)-\sum_{s} p_{s}\left(x_{j}\right)$, where the index $e$ runs through all null-geodesics ending at $x_{j}$ and $s$ runs through all null-geodesics starting at $x_{j}$.

Remark: The microlocal spectrum condition may be regarded as an analogue of the ususal spectrum condition in Minkowski spacetime.

The next requirement asks that the distributions $t_{n}$, defined in Eq. (10), have a certain scaling behaviour - roughly speaking, a certain "asymptotic degree of homogeneity" — at the total diagonal $\Delta_{n}(M)$ in $M^{n}$, which is compatible with the above microlocal spectrum condition. Mathematically, this can be expressed using the notion of the "microlocal scaling degree" $\mu \mathrm{sd}(t)$ of a distribution $t$, introduced in [3]. For a mathematically precise definition of this concept and some of its properties and related results, we refer to [3].

Microlocal scaling degree: The distributions $t_{n}$, defined in Eq. (10), have microlocal scaling degree

$$
\mu \operatorname{sd}\left(t_{n}\right) \leq \sum_{j} \operatorname{deg}\left(A_{j}\right)
$$

with respect to some cone $\Gamma_{n}$ (related to $\Gamma_{n}^{\mathscr{T}}$ ) at the total diagonal $\Delta_{n}(M)$. In other words, the scaling behaviour of the time ordered products has to follow what is usually called a "power counting rule".

Let us assume now that time ordered products with the above properties have been constructed up to order $n$. Then, by the causal factorization property, it can be seen that those of order $n+1$ are already given as (operator-valued) distributions on the space $M^{n+1} \backslash \Delta_{n+1}(M)$, i.e., away from the total diagonal. The remaining task is thus to extend these distributions to the entire space $M^{n+1}$. It turns out that this is indeed possible and that the extension can be performed in such a way that the so-defined time ordered products at order $n+1$ satisfy the requirements of causal factorization, permutation symmetry, unitarity, microlocal scaling degree and Wick expansion. However, the extension of these products to the total diagonal is in general not unique. Instead, there exist in general different extensions with the desired properties differing from each other by what is usually called a "finite renormalization ambiguity" 6 . The imposition of the above requirements on the time ordered products implies that this ambiguity must have a specific form. Firstly, by the Wick expansion property, the ambiguity in extending the time ordered products

\footnotetext{
${ }^{6}$ Of course finite renormalization ambiguities appear, in different guise, also in all other renormalization procedures.
} 
to the total diagonal is reduced to an ambiguity in extending the $\mathbb{C}$-number distributions $t_{n+1}$. The scaling degree requirement then further reduces that ambiguity to the choice of some set of smooth tensor fields on $M$. In order to make the latter statement more precise, we find it convenient to first introduce the notion of a "symbol". Let us denote points in $T_{y}^{*} M \times \cdots \times T_{y}^{*} M(n$ copies $)$ by $(y, \underline{k})=\left(y, k_{1}, \ldots, k_{n}\right)$.

Definition 2.1. A "symbol" $b$ is a map of the form

$$
b(y, \underline{k})=\sum_{m=0}^{\rho} \sum_{\pi \in \mathbf{P}(m, n)} b_{\pi}^{a_{1} \ldots a_{m}}(y) k_{\pi(1) a_{1}} \ldots k_{\pi(m) a_{m}},
$$

where $\mathbf{P}(m, n)$ is the set of all maps $\pi$ of the set $\{1, \ldots, m\}$ to the set $\{1, \ldots, n\}$, and where the $b_{\pi}^{a_{1} \ldots a_{m}}$ are smooth tensor fields of rank $m$ over $M$. The $\operatorname{order} \operatorname{deg}(b)$ of a symbol is defined as the maximum rank of all nonzero tensor fields appearing in the above expression. The principal part $\sigma_{b}(y, \underline{k})$ of a symbol $b$ is by definition the symbol obtained by collecting all terms in the above expression which contain nonzero tensor fields of rank $\operatorname{deg}(b)$.

Any symbol $b$ defines a distribution $B \in \mathscr{D}^{\prime}\left(M^{n+1}\right)$ by the formula

$$
B(y, \underline{x})=b(y,-i \underline{\nabla}) \delta(y, \underline{x}) .
$$

Here, we have used the abbreviations $\underline{\nabla}=\left(\nabla^{x_{1}}, \ldots, \nabla^{x_{n}}\right), \underline{x}=\left(x_{1}, \ldots, x_{n}\right)$ as well as $\delta(y, \underline{x})=\prod_{j=1}^{n} \delta\left(y, x_{j}\right)$ with $\delta$ the covariant delta function on $M$. Conversely, any distribution $B$ supported on the total diagonal in $M^{n+1}$ and with microlocal scaling degree $\mu \operatorname{sd}(B)<\infty$ at the diagonal arises in the above form for a symbol $b$ of degree $\leq \mu \mathrm{sd}(B)-4 n$.

Now 3] showed that the imposition of the Wick expansion and the scaling requirements reduce the ambiguity in defining $t_{n+1}\left(y, x_{1}, \ldots, x_{n}\right)$ to a distribution supported on the total diagonal with a microlocal scaling degree given by Eq. (111). Therefore, by what we just said, it must be of the form Eq. (12), where $b$ is some symbol of degree $\leq \mu \operatorname{sd}\left(t_{n+1}\right)-4 n$, whose form is further constrained by the unitarity and symmetry requirements. In a renormalizable theory, the maximum microlocal scaling degree of the distributions $t_{n+1}$ appearing in the expansion for the time ordered products, Eq. (9), does not increase with the order $n$ in perturbation theory. In the case at hand, these are the ones for which the interaction Lagrangian $\mathscr{L}$ has degree less or equal than four.

In this work, we need to impose a further normalization condition on the time ordered products (A proof of this for Minkowski space is given in [16], it can be adapted to curved spacetimes): 


\section{Equations of motion:}

$$
\begin{aligned}
\left(\square_{\mathbf{g}}-m^{2}\right)_{y} \mathscr{T}\left(A_{1}\left(x_{1}\right) \ldots A_{n}\left(x_{n}\right) \Phi(y)\right) & \\
\quad & i \sum_{j=1}^{n} \delta\left(y, x_{j}\right) \mathscr{T}\left(A_{1}\left(x_{1}\right) \ldots \frac{\partial A_{j}}{\partial \Phi}\left(x_{j}\right) \ldots A_{n}\left(x_{n}\right)\right),
\end{aligned}
$$

where we assume here, as will always be the case in the following, that the $A_{j}$ contain no derivatives. This condition ensures that the interacting field $\Phi_{\mathscr{L}}$ satisfies the non-linear equations of motion, in the sense that

$$
\left(\square_{\mathbf{g}}-m^{2}\right) \Phi_{g \mathscr{L}}(x)=-g(\partial \mathscr{L} / \partial \Phi)_{g \mathscr{L}}(x), \quad \text { for all } g \in \vartheta(\mathcal{O}) \text { and } x \in \mathcal{O} .
$$

\section{Local implementation of spacetime symmetries for self-interacting theories on curved spacetime by Noether charges}

Let us suppose now that $\mathbf{g}_{a b}$ admits a nowhere vanishing Killing vectorfield $\xi^{a}$, i.e. $£_{\xi} \mathbf{g}_{a b}=$ $\nabla_{(a} \xi_{b)}=0$, and let us denote by $\psi^{t}: M \rightarrow M$ the flow generated by that Killing field. The flow $\psi^{t}$ naturally induces a 1-parameter group of automorphisms $\alpha_{t}$ on the algebra $\mathfrak{A}$ of free quantum fields on $M$ by $\alpha_{t}(\Phi(f))=\Phi\left(f \circ \psi^{-t}\right)$. Let us make the further assumption that there exists a quasifree Hadamard state $\omega$ on $\mathfrak{A}$ for which $\omega\left(\alpha_{t}(a)\right)=\omega(a)$ for all $a \in \mathfrak{A}, t \in \mathbb{R}$, or equivalently, one for which

$$
\psi^{t *} \omega^{(2)}=\omega^{(2)} \text { for all } t \in \mathbb{R} .
$$

Then there is a strongly continuous 1-parameter group of unitaries $\{U(t)\}_{t \in \mathbb{R}}$ (with generator $H$ ) on the GNS-Hilbertspace $\mathscr{H}_{\omega}$ which generates this flow on the field operators (in the GNS-representation corresponding to $\omega$ ), i.e., $\Phi\left(f \circ \psi^{-t}\right)=U(t) \Phi(f) U(t)^{+}$for all $f \in \mathscr{D}(M)$.

\subsection{The action of the symmetry group on the algebra of inter- acting fields}

Let us assume that the interaction Lagrangian $\mathscr{L}$ is invariant under the spacetime symmetry, i.e., $\mathscr{L}\left(f \circ \psi^{-t}\right)=U(t) \mathscr{L}(f) U(t)^{+}$for all $f \in \mathscr{D}(M)$. Then it is possible to define an action of the 1-parameter group of spacetime symmetries $\left\{\psi^{t}\right\}_{t \in \mathbb{R}}$ by ${ }^{*}$-automorphisms $\alpha_{\mathscr{L}, t}$ on the algebra of interacting fields which satisfy

$$
\alpha_{\mathscr{L}, t}\left(\mathfrak{A}_{\mathscr{L}}(\mathcal{O})\right)=\mathfrak{A}_{\mathscr{L}}\left(\psi^{t} \mathcal{O}\right)
$$


for all bounded open regions $\mathcal{O} \subset M$, where $\psi^{t} \mathcal{O}$ denotes the transported region. In order to construct these automorphisms, we need to impose a further normalization condition.

Covariance: Let $A_{j}, j=1, \ldots, n$ be Wick monomials. Then we demand that

$$
\mathscr{T}\left(A_{1}\left(\psi^{t}\left(x_{1}\right)\right) \ldots A_{n}\left(\psi^{t}\left(x_{n}\right)\right)\right)=U(t) \mathscr{T}\left(A_{1}\left(x_{1}\right) \ldots A_{n}\left(x_{n}\right)\right) U(t)^{+} .
$$

Remark: We will not give a full proof here that the covariance requirement can indeed be satisfied, but rather only sketch the main arguments. Firstly, the multilocal Wick products : $\Phi^{\alpha_{1}}\left(x_{1}\right) \ldots \Phi^{\alpha_{n}}\left(x_{n}\right):_{\omega}$ by definition satisfy the covariance requirement, since the free field transforms as $\Phi\left(\psi^{t}(x)\right)=U(t) \Phi(x) U(t)^{+}$and since the two-point function $\omega^{(2)}$ is by assumption invariant under $\psi^{t}$. In view of the expansion property, covariance of the time ordered products is therefore equivalent to $\psi^{t *} t_{n}=t_{n}$ for all $\mathbb{C}$-number distributions $t_{n}$ of the form Eq. (10). As described in the preceeding section, the distributions $t_{n}$ at a given order $n$ are obtained by an extension procedure from those at order $<n$. The crucial ingredient in that extension procedure [3] is a projection operator $W_{n}: \mathscr{D}\left(M^{n}\right) \rightarrow \mathscr{D}\left(M^{n}\right)$ which maps any given testfunction to a testfunction that vanishes on the total diagonal $\Delta_{n}(M)$ together with a fixed number of its derivatives (that number is actually equal to the singular degree of $t_{n}$, given by $\left.\mu \mathrm{sd}\left(t_{n}\right)-4(n-1)\right)$. In order to prove the existence of an extension $t_{n}$ which is invariant under $\psi^{t}$, it is therefore sufficient to prove that there is a $\psi^{t}$-invariant choice for $W_{n}$ for all $n$, i.e., a choice for $W_{n}$, which commutes with the action of $\psi^{t *}$ on testfunctions, $W_{n} \circ \psi^{t *}=\psi^{t *} \circ W_{n}$. The crucial ingredient in the construction of $W_{n}$ (which potentially threatens the validity of this equation) is some bump function $w \in \mathscr{D}(M \times M)$ which is equal to one in a neighbourhood of $\Delta_{2}(M)$. Now, using the fact that $\xi^{a}$ is by definition nowhere vanishing, one can construct a bump function $w$ which is invariant under $\psi^{t}$, i.e., one for which $\psi^{t *} w=w$. It is not difficult to see that one can obtain from this a $W_{n}$ which is invariant under $\psi^{t}$, leading thus to invariant distributions $t_{n}$ at every order in perturbation theory.

Now, given an element $a \in \mathfrak{A}_{\mathscr{L}}(\mathcal{O})$, i.e., a family $a=\left(a_{g}\right)_{g \in \vartheta(\mathcal{O})}$ with $a_{g} \in \mathfrak{A}_{g \mathscr{L}}(\mathcal{O})$ and the property that $V a_{g^{\prime}}=a_{g} V$ for all $V \in \mathscr{V}_{g, g^{\prime}}(\mathcal{O})$, we define a family $\left(\alpha_{\mathscr{L}, t}(a)_{g}\right)_{g \in \vartheta\left(\psi^{t} \mathcal{O}\right)}$ of elements $\alpha_{\mathscr{L}, t}(a)_{g} \in \mathfrak{A}_{g \mathscr{L}}\left(\psi^{t} \mathcal{O}\right)$ by

$$
\alpha_{\mathscr{L}, t}(a)_{g}=U(t) a_{g \circ \psi^{t}} U(t)^{+} .
$$

We first want to show that this map defines an element in $\mathfrak{A}_{\mathscr{L}}\left(\psi^{t} \mathcal{O}\right)$. This means that we have to verify that

$$
V \alpha_{\mathscr{L}, t}(a)_{g^{\prime}}=\alpha_{\mathscr{L}, t}(a)_{g} V
$$

for all $V \in \mathscr{V}_{g, g^{\prime}}\left(\psi^{t} \mathcal{O}\right)$. A moment of reflection shows that this is the case if $V(t):=$ $U(t)^{+} V U(t)$ can be shown to be in the intertwiner space $\mathscr{V}_{g \circ \psi^{t}, g^{\prime} \circ \psi^{t}}(\mathcal{O})$. In order to see 
the latter, let us take an arbitrary Wick polynomial $A$ and an $f \in \mathscr{D}(\mathcal{O})$. Then, from the covariance requirement and the invariance of $\mathscr{L}$, we get

$$
\begin{aligned}
V(t) A_{g \circ \psi^{t} \mathscr{L}}(f) & =V(t) U(t)^{+} A_{g \mathscr{L}}\left(f \circ \psi^{-t}\right) U(t) \\
& =U(t)^{+} V A_{g \mathscr{L}}\left(f \circ \psi^{-t}\right) U(t) \\
& =U(t)^{+} A_{g^{\prime} \mathscr{L}}\left(f \circ \psi^{-t}\right) V U(t) \\
& =A_{g^{\prime} \circ \psi^{t} \mathscr{L}}(f) V(t),
\end{aligned}
$$

where in the second line we have used the expression (44) for the interacting field operators in terms of totally retarded products. Therefore $\alpha_{\mathscr{L}, t}(a)$ is a well-defined element in $\mathfrak{A}\left(\psi^{t} \mathcal{O}\right)$. It is clear from its definition that $\alpha_{\mathscr{L}, t}$ respects the product and ${ }^{*}$-operation in $\mathfrak{A}_{\mathscr{L}}(\mathcal{O})$ and that $\alpha_{\mathscr{L}, t+t^{\prime}}=\alpha_{\mathscr{L}, t} \circ \alpha_{\mathscr{L}, t^{\prime}}$. We have thus defined a 1-parameter group of automorphism as claimed.

Clearly, if there is no self-interaction, $\mathscr{L}=0$, the automorphism $\alpha_{\mathscr{L}, t}$ coincides with the above defined automorphism $\alpha_{t}$ on the algebras of free fields. By definition, we also have that

$$
\alpha_{\mathscr{L}, t}\left(A_{\mathscr{L}}(f)\right)=A_{\mathscr{L}}\left(f \circ \psi^{-t}\right)
$$

for any interacting operator $A_{\mathscr{L}}(f)$ defined from a Wick polynomial $A$. The generator of the 1-parameter group $\{\alpha \mathscr{L}, t\}_{t \in \mathbb{R}}$ is given by the derivation

$$
\delta_{\mathscr{L}}(a)=i \frac{d}{d t} \alpha_{\mathscr{L}, t}(a)
$$

The action of that derivation on elements of the form $A_{\mathscr{L}}(f)$ it is given by $\delta_{\mathscr{L}}\left(A_{\mathscr{L}}(f)\right)=$ $-i A_{\mathscr{L}}\left(\xi^{a} \nabla_{a} f\right)$.

\subsection{Implementation of $\delta_{\mathscr{L}}$ by a local operator}

The aim of this subsection is to show that for any bounded region $\mathcal{O} \subset M$, and any bounded, open, globally hyperbolic region $\widehat{\mathcal{O}}$ strongly containing $\mathcal{O}$, there exists a charge operator $Q_{\mathscr{L}}$ contained in $\mathfrak{A}_{\mathscr{L}}(\widehat{\mathcal{O}})$, which implements the infinitesimal action $\delta_{\mathscr{L}}$ of the spacetime symmetries on $\mathfrak{A}_{\mathscr{L}}(\mathcal{O})$,

$$
\delta_{\mathscr{L}}(a)=\left[Q_{\mathscr{L}}, a\right], \quad \text { for all } a \in \mathfrak{A}_{\mathscr{L}}(\mathcal{O}),
$$

and which arises, in some sense, as a surface-integral of an interacting Noether currentdensity corresponding to the symmetry $\xi^{a}$. As a preparation, we first consider the free-field case (i.e., $\mathscr{L}=0$ ). 


\subsubsection{Free fields}

We consider the covariantly conserved ${ }^{7}$ free current-density $j^{a}=\Theta^{a b} \xi_{b}$, where

$$
\Theta_{a b}=: \nabla_{a} \Phi \nabla_{b} \Phi-\frac{1}{2} \mathbf{g}_{a b}\left(\nabla^{c} \Phi \nabla_{c} \Phi+m^{2} \Phi^{2}\right): \omega
$$

We now construct from $j^{a}$ a free charge operator $Q$ in $\mathfrak{A}(\widehat{\mathcal{O}})$, which implements the infinitesimal action of the symmetry on $\mathfrak{A}(\mathcal{O})$. In order to do so, we choose the following

\section{"Technical data":}

- A Cauchy surface $\widehat{\Sigma}$ in $\widehat{\mathcal{O}}$ and Gaussian coordinates around $\widehat{\Sigma}$, described by the map $[-\epsilon, \epsilon] \times \widehat{\Sigma} \ni(t, x) \mapsto \operatorname{Exp}_{x} t n^{a} \in M$, where $n^{a}$ is the surface normal of $\widehat{\Sigma}$. (Note that this implies that the vectorfield $n^{a}=(\partial / \partial t)^{a}$ satisfies $n^{a} n_{a}=-1, n^{a} \nabla_{a} n^{b}=0$ and $\nabla_{[a} n_{b]}=0$ near $\widehat{\Sigma}$.)

- An open subset $\Sigma \subset \widehat{\Sigma}$ such that $\mathcal{O} \subset D(\Sigma) \subset \widehat{\mathcal{O}}$ and a function $f \in \mathscr{D}(\widehat{\mathcal{O}})$ which can be written as $f(x)=\rho(t(x))$ on a neighbourhood of $J(\mathcal{O}) \cap \widehat{\mathcal{O}}$, where $\rho$ is a smooth, real valued function with compact support on $[-\epsilon, \epsilon]$ such that $\int \rho\left(x^{0}\right) \mathrm{d} x^{0}=1$, and $\epsilon$ is chosen so small that $\operatorname{Exp}_{x} t n^{a}$ is contained in $\widehat{\mathcal{O}}$ for all $x \in \Sigma$ and $|t| \leq \epsilon$. (Note that this implies that $h_{b}^{a} \nabla_{a} f=0$ on $J(\mathcal{O}) \cap \widehat{\mathcal{O}}$, where $h^{a b}=\mathbf{g}^{a b}+n^{a} n^{b}$.)

We have the following Lemma:

Lemma 3.1. The operator

$$
Q=j^{a}\left(n_{a} f\right)=\Theta^{a b}\left(n_{a} \xi_{b} f\right)
$$

generates the Killing symmetry on all observables localized in $\mathcal{O}, \delta(a)=[Q, a]$ for all $a \in \mathfrak{A}(\mathcal{O})$ and for any $f, n^{a}$ as in "technical data". In other words,

$$
[Q, \Phi(x)]=i \xi^{a} \nabla_{a} \Phi(x) \quad \text { for all } x \in \mathcal{O} .
$$

Remark: Note that $Q$ is morally given by an surface integral over a Cauchy surface of the time-component of $j^{a}$. This is clear because the function $f(x)$ is of the form $\rho(t(x))$ on $J(\mathcal{O}) \cap \widehat{\mathcal{O}}$ for some bump function $\rho$, which one can imagine to be given by an "infinitely sharp spike".

Proof. One computes

$$
\begin{aligned}
{[Q, \Phi(x)] } & =\int_{M}\left[\Theta_{a b}(y), \Phi(x)\right] f n^{a} \xi^{b}(y) \mu_{\mathbf{g}}(y) \\
& =i \int_{J(\mathcal{O})}\left(f n^{(a} \xi^{b)}(y) \nabla_{a} \Phi(y) \nabla_{b}^{y} \Delta(x, y)+\nabla^{a}\left(f n^{a} \xi_{b}\right)(y) \Phi(y) \nabla_{a}^{y} \Delta(x, y)\right) \mu_{\mathbf{g}}(y)
\end{aligned}
$$

\footnotetext{
${ }^{7}$ It follows from the equations of motion that $\nabla^{a} \Theta_{a b}=0$, therefore $\nabla_{a} j^{a}=\nabla_{a} \Theta^{a b} \xi_{b}+\Theta^{a b} \nabla_{(a} \xi_{b)}=0$.
} 
where in the second line we have performed a partial integration and used the equations of motion. On the other hand

$$
\begin{aligned}
i \xi^{a} \nabla_{a} \Phi(x) & =i \int_{\Sigma} \Phi(y) \stackrel{\leftrightarrow}{\nabla_{a}^{y}} \xi^{b} \nabla_{b}^{x} \Delta(x, y) \mathrm{d} S^{a}(y) \\
& =i \int_{J(\mathcal{O})}\left(\Phi(y) \stackrel{\leftrightarrow}{\nabla}_{a}^{y} \xi^{b} \nabla_{b}^{x} \Delta(x, y)\right) n^{a} f(y) \mu_{\mathbf{g}}(y) \\
& =-i \int_{J(\mathcal{O})}\left(\Phi(y) \stackrel{\leftrightarrow}{\nabla_{a}^{y}}\left(\xi^{b} \nabla_{b}^{y} \Delta(x, y)\right)\right) n^{a} f(y) \mu_{\mathbf{g}}(y)
\end{aligned}
$$

where in the second line we have used that $\xi^{a} \nabla_{a}^{x} \Delta(x, y)=-\xi^{a} \nabla_{a}^{y} \Delta(x, y)$, which holds because $\xi^{a}$ is a Killing field. This gives

$$
\begin{aligned}
i \xi^{a} \nabla_{a} \Phi(x)=i \int_{J(\mathcal{O})}\left(f n^{(a} \xi^{b)}(y)\right. & \nabla_{a} \Phi(y) \nabla_{b}^{y} \Delta(x, y) \\
& \left.-\left(f n^{b} \nabla_{b} \xi^{a}(y)-\nabla_{b}\left(f n^{a} \xi^{b}\right)(y)\right) \Phi(y) \nabla_{a}^{y} \Delta(x, y)\right) \mu_{\mathbf{g}}(y),
\end{aligned}
$$

So, in order to prove the lemma, we must show that

$$
\nabla_{b}\left(f n^{a} \xi^{b}\right)-f n^{b} \nabla_{b} \xi^{a}=\nabla^{a}\left(f n^{b} \xi_{b}\right)
$$

on $J(\mathcal{O}) \cap \widehat{\mathcal{O}}$. The left side of this equation is equal to

$$
\begin{aligned}
\text { l.s. } & =f n^{a} \nabla_{b} \xi^{b}+\left(\xi^{b} \nabla_{b} f\right) n^{a}+\xi^{b} \nabla_{b} n^{a} f-n^{b} \nabla_{b} \xi^{a} f \\
& =\left(\xi^{b} \nabla_{b} f\right) n^{a}+[\xi, n]^{a} f \\
& =n^{a} n^{c} \nabla_{c} f n^{b} \xi_{b}+n^{a} \xi^{c} h_{c}{ }^{b} \nabla_{b} f+[\xi, n]^{a} f \\
& =n^{a} n^{c} \nabla_{c} f n^{b} \xi_{b}+[\xi, n]^{a} f,
\end{aligned}
$$

where we have used that $\nabla_{a} \xi^{a}=0$ by Killing's equation. The hand side is given by

$$
\begin{aligned}
\text { r.s. } & =\nabla^{a} f n^{b} \xi_{b}+f\left(\xi_{b} \nabla^{a} n^{b}+n_{b} \nabla^{a} \xi^{b}\right) \\
& =\nabla^{a} f n^{b} \xi_{b}+[\xi, n]^{a} f \\
& =n^{a} n^{c} \nabla_{c} f n^{b} \xi_{b}+[\xi, n]^{a} f,
\end{aligned}
$$

where we have used that $\nabla^{(a} \xi^{b)}=0, \nabla^{[a} n^{b]}=0$ and that $h^{a}{ }_{b} \nabla^{b} f=0$ on $J(\mathcal{O}) \cap \widehat{\mathcal{O}}$. Hence both sides are equal, thus proving the lemma.

\subsubsection{Interacting fields}

Let $f$ be a function of compact support in $\widehat{\mathcal{O}}$ and $n^{a}$ a timelike vectorfield, with the properties described in the previous subsection under "technical data". For any function $g$ in $\vartheta(\widehat{\mathcal{O}})$ we define an interacting current density by

$$
J_{g \mathscr{L}}^{a}=j_{g \mathscr{L}}^{a}+g \xi^{a} \mathscr{L}_{g \mathscr{L}}=\left(\Theta_{g \mathscr{L}}^{a b}+g \mathbf{g}^{a b} \mathscr{L}_{g \mathscr{L}}\right) \xi_{b}
$$


where the operator $j_{g \mathscr{L}}^{a}$ is given by

$$
j_{g \mathscr{L}}^{a}\left(h_{a}\right)=S[g \mathscr{L}]^{-1} \frac{\partial}{i \partial t} S\left[g \mathscr{L}+t h_{a} j^{a}\right]=\Theta_{g \mathscr{L}}^{a b}\left(h_{a} \xi_{b}\right)
$$

with $j^{a}$ the free current density. By analogy with the free charge, we next define an interacting charge by

$$
Q_{g \mathscr{L}}=J_{g \mathscr{L}}^{a}\left(n_{a} f\right)
$$

Our main result is that the interacting current density $J_{\mathscr{L}}^{a}$ is conserved and the the corresponding charge operator $Q_{\mathscr{L}} \in \mathfrak{A}_{\mathscr{L}}(\widehat{\mathcal{O}})$ generates the symmetry induced by $\xi^{a}$ on the algebra $\mathfrak{A}_{\mathscr{L}}(\mathcal{O})$. We formulate this as a theorem:

Theorem 3.1. The interacting current density $J_{\mathscr{L}}^{a}$ is conserved,

$$
\nabla_{a} J_{g \mathscr{L}}^{a}(x)=0 \quad \text { for all } x \in \mathcal{O} \text { and } g \in \vartheta(\widehat{\mathcal{O}})
$$

in the sense of formal power series in the coupling constant $\lambda$. The interacting charge $Q_{\mathscr{L}}$ implements the infinitesimal action of the spacetime symmetry $\xi^{a}$ on interacting fields in the sense that $\delta_{\mathscr{L}}(a)=\left[Q_{\mathscr{L}}, a\right]$ for all $a \in \mathfrak{A}_{\mathscr{L}}(\mathcal{O})$. In other words

$$
\left[Q_{g \mathscr{L}}, A_{g \mathscr{L}}(x)\right]=i \xi^{a} \nabla_{a} A_{g \mathscr{L}}(x) \quad \text { for all } x \in \mathcal{O} \text { and } g \in \vartheta(\widehat{\mathcal{O}})
$$

and for any Wick polynomial $A$. (This holds for any choice of function $f$ and timelike vectorfield $n^{a}$ with the properties described in the previous section under "technical data".)

Proof. The proof of the above result is divided into two parts. In the first part, we show that the time ordered products can be normalized in such a way that the interacting current density $J_{g \mathscr{L}}^{a}$ is covariantly conserved. A proof of this requires the demonstration of a corresponding set of Ward identities, see Eq. (20) below. A proof of a similar set of Ward identities for the stress energy tensor in Minkowski space was previously given independently by [13] and 9]. Our proof of the Ward identities follows [13] and 9] closely, up to the point at which one has to remove the anomaly. Here the methods of the present paper differ from those of [13] and [9], which are based on momentum space techniques and therefore not suitable in the present context. In the second part we then demonstrate (by a chain of arguments somewhat similar to the one given in [13, pp 50-51]) that conservation of the interacting current implies the second statement of the theorem, Eq. (19).

In order to show conservation of the interacting current, we first expand $J_{g \mathscr{L}}^{a}$ in terms of totally retarded products (cf. Eq. (4)), and express these by products of time ordered 
products (cf. Eq. (5)). It is then not difficult to see that Eq. (18) is equivalent to the set of Ward identities

$$
\nabla_{a}^{y} \mathscr{T}\left(j^{a}(y) A_{1}\left(x_{1}\right) \ldots A_{n}\left(x_{n}\right)\right)=i \sum_{j=1}^{n} \delta\left(y, x_{j}\right) \xi^{a} \nabla_{a}^{x_{j}} \mathscr{T}\left(A_{1}\left(x_{1}\right) \ldots \ldots A_{n}\left(x_{n}\right)\right)
$$

for all $n=1,2, \ldots$ and all possible sub-Wick monomials $A_{j}, j=1, \ldots, n$ of $\mathscr{L}$ (which therefore do not contain derivatives). A proof of the Ward identities, Eq. (20), is given in the Appendix.

We now show that the Ward identities imply Eq. (19). Assume first that $J^{-}(x) \cap$ $\operatorname{supp}(f)=\emptyset$. Then it is easy to see that there is a function $F \in \mathscr{D}(\widehat{\mathcal{O}})$ for which $\nabla^{a} F=$ $n^{a} f$ on some neighbourhood of $J^{+}(x) \cap \widehat{\mathcal{O}}$ and for which $F=1$ in some neighbourhood of $x$. We then have

$$
\begin{aligned}
& {\left[j_{g \mathscr{L}}^{a}\left(n_{a} f\right), A_{g \mathscr{L}}(x)\right]=\sum_{n=0}^{\infty} \frac{i^{n}}{n !} \int \mu_{\mathbf{g}}(\underline{x}) g\left(x_{1}\right) \ldots g\left(x_{n}\right) \times} \\
& \int \mu_{\mathbf{g}}(y) n^{a} f(y)\left(\mathscr{R}\left(j^{a}(y) ; A(x) \underline{\mathscr{L}}(\underline{x})\right)-\mathscr{R}\left(A(x) ; j^{a}(y) \underline{\mathscr{L}}(\underline{x})\right)\right),
\end{aligned}
$$

where we have used the abbreviation $\underline{L}(\underline{x})=\mathscr{L}\left(x_{1}\right) \ldots \mathscr{L}\left(x_{n}\right)$. By the support properties of $f$ and the support properties of the totally retarded products, this is equal to

$$
=\sum_{n=0}^{\infty} \frac{i^{n}}{n !} \int \mu_{\mathbf{g}}(\underline{x}) \mu_{\mathbf{g}}(y) g\left(x_{1}\right) \ldots g\left(x_{n}\right) \nabla_{a} F(y) \mathscr{R}\left(j^{a}(y) ; A(x) \underline{L}(\underline{x})\right) .
$$

We next use the Ward identities for the $\mathscr{R}$-products (which are easily obtained from the Ward-identities Eq. (20) for the $\mathscr{T}$-products and formula Eq. (15). This gives

$$
\begin{aligned}
& =i \sum_{n=0}^{\infty} \frac{i^{n}}{n !} \int \mu_{\mathbf{g}}(\underline{x}) \mu_{\mathbf{g}}(y) g\left(x_{1}\right) \ldots g\left(x_{n}\right) F(y) \delta(y, x) \xi^{a} \nabla_{a}^{x} \mathscr{R}(A(x) ; \mathscr{L}(\underline{x})) \\
& +i \sum_{n=1}^{\infty} \frac{i^{n}}{n !} \int \mu_{\mathbf{g}}(\underline{x}) \mu_{\mathbf{g}}(y) g\left(x_{1}\right) \ldots g\left(x_{n}\right) F(y) \sum_{j=1}^{n} \delta\left(y, x_{j}\right) \xi^{a} \nabla_{a}^{x_{j}} \mathscr{R}\left(\mathscr{L}\left(x_{j}\right) ; A(x) \underline{L} \backslash j(\underline{x})\right),
\end{aligned}
$$

where $\mathscr{L} \backslash j(\underline{x})=\mathscr{L}\left(x_{1}\right) \ldots \mathscr{L}\left(x_{j}\right) \ldots \mathscr{L}\left(x_{n}\right)$. We observe that the first sum is just $i \xi^{a} \nabla_{a} A_{g \mathscr{L}}(x)$. Performing the $x_{j^{-}}$integrations in the second term, using that $\nabla^{a} F=n^{a} f$ on a neighbourhood of $J^{+}(x) \cap \widehat{\mathcal{O}}$, and observing that the $y$-support of $\mathscr{R}(\mathscr{L}(y) ; A(x) \ldots)$ is contained in $J^{+}(x) \cap \widehat{\mathcal{O}}$, we find that the above expression is equal to

$$
\begin{aligned}
= & i \xi^{a} \nabla_{a} A_{g} \mathscr{L}(x) \\
- & \sum_{n=1}^{\infty} \frac{i^{n-1}}{n !} \sum_{j=1}^{n} \int \mu_{\mathbf{g}}\left(x_{1}\right) \ldots \mu_{\mathbf{g}}\left(x_{j}\right) \ldots \mu_{\mathbf{g}}\left(x_{n}\right) \times \\
& g\left(x_{1}\right) \ldots g\left(x_{j}\right) \ldots g\left(x_{n}\right) n_{a} f(y) \mathscr{R}\left(g \xi^{a} \mathscr{L}(y) ; A(x) \underline{L} \backslash j(\underline{x})\right) .
\end{aligned}
$$


But the terms under the sum over $j$ are all equal, by the symmetry of the $\mathscr{R}$-products, therefore this expression is (we shift the first summation index)

$$
\begin{aligned}
& =i \xi^{a} \nabla_{a} A_{g} \mathscr{L}(x) \\
& -\sum_{n=0}^{\infty} \frac{i^{n}}{n !} \int \mu_{\mathbf{g}}(\underline{x}) g\left(x_{1}\right) \ldots g\left(x_{n}\right) n_{a} f(y) \mathscr{R}\left(g \xi^{a} \mathscr{L}(y) ; A(x) \underline{L}(\underline{x})\right) .
\end{aligned}
$$

Now, again using that $\operatorname{supp}(f) \cap J^{-}(x)=\emptyset$ and the support properties of the totally retarded products, we see that we may add the term $\mathscr{R}\left(A(x) ; g \xi^{a} \mathscr{L}(y) \underline{L}(\underline{x})\right)$ under the integral, because this does not make any contribution. This then makes it obvious that the above expression is just

$$
=i \xi^{a} \nabla_{a} A_{g} \mathscr{L}(x)-\left[g \xi^{a} \mathscr{L}_{g \mathscr{L}}\left(n_{a} f\right), A_{g \mathscr{L}}(x)\right]
$$

which proves Eq. (19) if $\operatorname{supp}(f) \cap J^{-}(x)=\emptyset$. By a similar chain of arguments, one can prove that Eq. (19) also holds if instead $\operatorname{supp}(f) \cap J^{+}(x)=\emptyset$. We will now show that the general case follows from these two facts. To this end, we will show that, for an arbitrary $f$ as in "technical data", one can always construct a function $\widehat{f}$ with the same properties as $f$ and with the additional properties that: $\operatorname{supp}(\widehat{f}) \subset \operatorname{supp}(f)$, there holds either that $J^{+}(x) \cap \operatorname{supp}(\widehat{f})=\emptyset$ or $J^{-}(x) \cap \operatorname{supp}(\widehat{f})=\emptyset$ and

$$
(f-\widehat{f}) n^{a}=\nabla^{a} F
$$

in a neighbourhood of $J(x) \cap \widehat{\mathcal{O}}$, where $F \in \mathscr{D}(\widehat{\mathcal{O}})$. We may thus write

$$
\begin{aligned}
{\left[J_{g \mathscr{L}}^{a}\left(n_{a} f\right), A_{g \mathscr{L}}(x)\right] } & =\left[J_{g \mathscr{L}}^{a}\left(n_{a} \widehat{f}\right), A_{g \mathscr{L}}(x)\right]+\left[J_{g \mathscr{L}}^{a}\left(n_{a}(f-\widehat{f})\right), A_{g} \mathscr{L}(x)\right] \\
& =i \xi^{a} \nabla_{a} A_{g \mathscr{L}}(x)+\left[J_{g \mathscr{L}}^{a}\left(\nabla_{a} F\right), A_{g \mathscr{L}}(x)\right]
\end{aligned}
$$

where in the last line we have used the fact that we already know Eq. (19) for functions like $\widehat{f}$. This then proves the theorem, because $J_{g \mathscr{L}}^{a}\left(\nabla_{a} F\right)=0$, by current conservation.

It thus remains to construct an $\widehat{f}$ as in "technical data" in the previous subsection, such that in addition Eq. (21) holds. Now recall that $f$ is of the form $f(x)=\rho(t(x))$ in a neighbourhood of $J(\mathcal{O}) \cap \widehat{\mathcal{O}}$ where $\rho$ is a compactly supported smooth function on $[-\epsilon, \epsilon]$ such that $\int \rho\left(y^{0}\right) \mathrm{d} y^{0}=1$. Let us choose a $\widehat{\rho}$ with $\operatorname{supp}(\widehat{\rho}) \subset \operatorname{supp}(\rho) \operatorname{such}$ that either $t(x)>\sup \operatorname{supp}(\widehat{\rho})$ or $t(x)<\inf \operatorname{supp}(\widehat{\rho})$, and a function $\widehat{f} \in \mathscr{D}(\widehat{\mathcal{O}})$ satisfying $\widehat{f}(x)=\widehat{\rho}(t(x))$ on $J(\mathcal{O}) \cap \widehat{\mathcal{O}}$. This function then clearly satisfies either $\operatorname{supp}(\widehat{f}) \cap J^{+}(x)=0$ or $\operatorname{supp}(\widehat{f}) \cap J^{+}(x)=0$. Let us define

$$
F(y)=\int_{-\infty}^{t(y)} \rho\left(y^{0}\right)-\widehat{\rho}\left(y^{0}\right) \mathrm{d} y^{0}
$$


for $y \in J(\mathcal{O}) \cap \widehat{\mathcal{O}}$. It is clear that $F$ can be continued smoothly to a function in $\mathscr{D}(\widehat{\mathcal{O}})$. By definition of $F$, Eq. (21) holds in a neighbourhood of $J(x) \cap \widehat{\mathcal{O}}$. We have thus constructed a $\widehat{f}$ with the desired properties, thus finishing the proof.

Acknowledgements: I would like to thank K. Fredenhagen for suggesting to me the idea for the proof of Eq. (14), as well as for pointing out several errors in earlier versions of this manuscript. This work was supported by NSF-grant PHY00-90138 to the University of Chicago.

\section{Appendix: Proof of the Ward identities, Eq. (201)}

Proof. We want to prove the Ward identities by an induction on $n$ and the total degree of the Wick monomials defined by $\omega:=\sum_{j=1}^{n} \operatorname{deg}\left(A_{j}\right)$. Let us write $D\left(y, x_{1}, \ldots, x_{n}\right)$ for the left hand side minus the right hand side of Eq. (20), i.e., the anomaly and let us inductively assume that the Ward identities can be satisfied for some $n$ and some order $\omega$. The logic of the induction step is the following. In Step 1), we show that the Ward identities for $\omega+1$ and $n$, can be reduced to the scalar identity obtained by taking the vacuum expectation value of Eq. (20). It is argued in Step 3) that these can be satisfied. By what we have just said, it is then sufficient to show the Ward identities for $(n+1), \rho$, when one of the $A_{j}$ is equal to $\Phi$. Again, arguing as in Step 1), only the scalar identity has to be proven. This is done in Step 2).

Step 1: We want to show that $D\left(y, x_{1}, \ldots, x_{n}\right)$ is a multiple of the identity operator. To show this, we first demonstrate that it commutes with any free field operator. We have

$$
\begin{aligned}
& {[D(y, \underline{x}), \Phi(z)]=\nabla_{a}^{y} \mathscr{T}\left(\left[j^{a}(y), \Phi(z)\right] A_{1}\left(x_{1}\right) \ldots A_{n}\left(x_{n}\right)\right)+} \\
& i \sum_{j=1}^{n} \Delta\left(x_{j}, z\right) \nabla_{a}^{y} \mathscr{T}\left(j^{a}(y) \ldots \frac{\partial A_{j}}{\partial \Phi}\left(x_{j}\right) \ldots A_{n}\left(x_{n}\right)\right)+ \\
& i \sum_{j=1}^{n} \delta\left(y, x_{j}\right) \xi^{a} \nabla_{a}^{x_{j}} \sum_{k=1}^{n} \Delta\left(x_{k}, z\right) \mathscr{T}\left(A_{1}\left(x_{1}\right) \ldots \frac{\partial A_{k}}{\partial \Phi}\left(x_{k}\right) \ldots A_{n}\left(x_{n}\right)\right) .
\end{aligned}
$$

To proceed, we calculate

$$
\left[j^{a}(y), \Phi(z)\right]=i\left(\nabla^{(a} \Phi(y) \nabla^{b)} \Delta(y, z)-\mathbf{g}^{a b}\left(\nabla^{c} \Phi(y) \nabla_{c} \Delta(y, z)+m^{2} \Phi(y) \Delta(y, z)\right)\right) \xi_{b}(y) .
$$


We now demand that time ordered products containing a once differentiated free-field factor satisfy the following normalization condition $^{8}$

$$
\mathscr{T}\left(\nabla_{a} \Phi(y) A_{1}\left(x_{1}\right) \ldots A_{n}\left(x_{n}\right)\right)=\nabla_{a}^{y} \mathscr{T}\left(\Phi(y) A_{1}\left(x_{1}\right) \ldots A_{n}\left(x_{n}\right)\right)
$$

The above expression for $\left[j^{a}(y), \Phi(z)\right]$ together with the normalization condition (N4) then gives:

$$
\begin{aligned}
\nabla_{a}^{y} \mathscr{T}\left(\left[j^{a}(y), \Phi(z)\right]\right. & \left.A_{1}\left(x_{1}\right) \ldots A_{n}\left(x_{n}\right)\right)= \\
& i \sum_{j=1}^{n} \delta\left(x_{j}, y\right) \xi^{a} \nabla_{a} \Delta(y, z) \mathscr{T}\left(A_{1}\left(x_{1}\right) \ldots \frac{\partial A_{j}}{\partial \Phi}\left(x_{j}\right) \ldots A_{n}\left(x_{n}\right)\right) .
\end{aligned}
$$

From this, we obtain

$$
\begin{aligned}
{[D(y, \underline{x}), \Phi(z)]=i \sum_{j=1}^{n} \Delta\left(x_{j}, z\right) } & \left\{\nabla_{a}^{y} \mathscr{T}\left(j^{a}(y) \ldots \frac{\partial A_{j}}{\partial \Phi}\left(x_{j}\right) \ldots A_{n}\left(x_{n}\right)\right)\right. \\
& \left.-i \sum_{k=1}^{n} \delta\left(y, x_{k}\right) \xi^{a} \nabla_{a}^{x_{k}} \mathscr{T}\left(A_{1}\left(x_{1}\right) \ldots \frac{\partial A_{j}}{\partial \Phi}\left(x_{j}\right) \ldots A_{n}\left(x_{n}\right)\right)\right\} .
\end{aligned}
$$

Now the expression in braces vanishes by the Ward identities at total degree less than $\omega$, showing thus that $D$ commutes with the free field. By the Wick expansion requirement, the operator $D$ must be a linear combination of (multi-local) Wick products with distributional coefficients. Now it can be seen that any operator of this form which commutes with a free field is in fact a multiple of the identity, showing thus that $D$ is a $\mathbb{C}$-number.

We next want to show that the numerical distribution $D$ is localized at $x_{1}=\cdots=$ $x_{n}=y$. In order to see this, note that for any point $M^{n+1} \ni\left(x_{1}, \ldots, x_{n}, y\right) \neq(y, \ldots, y)$, one can find a Cauchy surface $\Sigma$ in $M$ which separates some points, $\left\{x_{j}\right\}_{j \in J, J \neq \emptyset}$ say, from the other points $\left\{x_{j}\right\}_{j \notin J}$ and $y$. Without loss of generality we assume that the latter are in not in the causal past of the first set of points. Then, by causal factorization and the induction hypothesis, we have

$$
D\left(y, x_{1}, \ldots, x_{n}\right)=\underbrace{D\left(y,\left\{x_{j}\right\}_{j \notin J}\right)}_{=0} \mathscr{T}\left(\prod_{j \in J} A_{j}\left(x_{j}\right)\right)=0 .
$$

Therefore we conclude $D(y, \underline{x})$ is a scalar distribution supported on the total diagonal in $M^{n+1}$.

\footnotetext{
${ }^{8}$ This condition is essentially equivalent to a generalization of (N4) to time ordered products containing a once differentiated free field factor. For a more detailed discussion, see [13, p. 52].
} 
Step 2: We show that the scalar Ward identities hold for $n+1$ factors when one of the Wick monomials is a free field. We find from the formula for the time ordered products with a free field factor, Eq. (N4), that

$$
\begin{aligned}
& \left\langle\Omega_{\omega} \mid \mathscr{T}\left(j^{a}(y) \Phi(x) A_{1}\left(x_{1}\right) \ldots A_{n}\left(x_{n}\right)\right) \Omega_{\omega}\right\rangle \\
= & i \sum_{j=1}^{n} \Delta_{F}\left(x, x_{j}\right)\left\langle\Omega_{\omega} \mid \mathscr{T}\left(j^{a}(y) A_{1}\left(x_{1}\right) \ldots \frac{\partial A_{j}}{\partial \Phi}\left(x_{j}\right) \ldots A_{n}\left(x_{n}\right)\right) \Omega_{\omega}\right\rangle \\
+ & \left.\left.i \xi_{b}(y) \nabla^{(b} \Delta_{F}(y, x)\left\langle\Omega_{\omega}\right| \mathscr{T}\left(\nabla^{a}\right) \Phi(y) A_{1}\left(x_{1}\right) \ldots A_{n}\left(x_{n}\right)\right) \Omega_{\omega}\right\rangle \\
- & i \xi^{a}(y)\left(\nabla_{b} \Delta_{F}(y, x)\left\langle\Omega_{\omega}, \mathscr{T}\left(\nabla^{b} \Phi(y) A_{1}\left(x_{1}\right) \ldots A_{n}\left(x_{n}\right)\right) \Omega_{\omega}\right\rangle\right. \\
+ & \left.m^{2} \Delta_{F}(y, x)\left\langle\Omega_{\omega} \mid \mathscr{T}\left(\Phi(y) A_{1}\left(x_{1}\right) \ldots A_{n}\left(x_{n}\right)\right) \Omega_{\omega}\right\rangle\right)
\end{aligned}
$$

where $\Delta_{F}=\omega^{(2)}+i \Delta_{A}$ is the Feynman propagator. From this one obtains

$$
\begin{aligned}
& \nabla_{a}^{y}\left\langle\Omega_{\omega} \mid \mathscr{T}\left(j^{a}(y) \Phi(x) A_{1}\left(x_{1}\right) \ldots A_{n}\left(x_{n}\right)\right) \Omega_{\omega}\right\rangle \\
= & i \sum_{j=1}^{n} \Delta_{F}\left(x, x_{j}\right) \nabla_{a}^{y}\left\langle\Omega_{\omega} \mid \mathscr{T}\left(j^{a}(y) A_{1}\left(x_{1}\right) \ldots \frac{\partial A_{j}}{\partial \Phi}\left(x_{j}\right) \ldots A_{n}\left(x_{n}\right)\right) \Omega_{\omega}\right\rangle \\
+ & i \xi^{a} \nabla_{a} \Delta_{F}(y, x)\left(\square_{\mathrm{g}}-m^{2}\right)_{y}\left\langle\Omega_{\omega} \mid \mathscr{T}\left(\Phi(y) A_{1}\left(x_{1}\right) \ldots A_{n}\left(x_{n}\right)\right) \Omega_{\omega}\right\rangle \\
+ & i \delta(y, x)\left\langle\Omega_{\omega} \mid \mathscr{T}\left(\xi^{a} \nabla_{a} \Phi(y) A_{1}\left(x_{1}\right) \ldots A_{n}\left(x_{n}\right)\right) \Omega_{\omega}\right\rangle .
\end{aligned}
$$

Now using condition (는 and the Ward identities for $n$ factors, one finds that this is equal to

$$
\begin{aligned}
& =-\sum_{j, k=1}^{n} \Delta_{F}\left(x, x_{j}\right) \delta\left(y, x_{k}\right) \xi^{a} \nabla_{a}^{x_{k}}\left\langle\Omega_{\omega} \mid \mathscr{T}\left(A_{1}\left(x_{1}\right) \ldots \frac{\partial A_{j}}{\partial \Phi}\left(x_{j}\right) \ldots A_{n}\left(x_{n}\right)\right) \Omega_{\omega}\right\rangle \\
& -\sum_{j=1}^{n} \xi^{a} \nabla_{a} \Delta_{F}(y, x) \delta\left(y, x_{j}\right)\left\langle\Omega_{\omega} \mid \mathscr{T}\left(\Phi(y) A_{1}\left(x_{1}\right) \ldots \frac{\partial A_{j}}{\partial \Phi}\left(x_{j}\right) \ldots A_{n}\left(x_{n}\right)\right) \Omega_{\omega}\right\rangle \\
& +i \delta(y, x) \xi^{a} \nabla_{a}^{y}\left\langle\Omega_{\omega} \mid \mathscr{T}\left(\Phi(y) A_{1}\left(x_{1}\right) \ldots A_{n}\left(x_{n}\right)\right) \Omega_{\omega}\right\rangle \\
& =i \sum_{j=1}^{n} \delta\left(y, x_{j}\right) \xi^{a} \nabla_{a}^{x_{j}}\left\langle\Omega_{\omega} \mid \mathscr{T}\left(\Phi(x) A_{1}\left(x_{1}\right) \ldots A_{n}\left(x_{n}\right)\right) \Omega_{\omega}\right\rangle \\
& +i \delta(y, x) \xi^{a} \nabla_{a}^{x}\left\langle\Omega_{\omega} \mid \mathscr{T}\left(\Phi(x) A_{1}\left(x_{1}\right) \ldots A_{n}\left(x_{n}\right)\right) \Omega_{\omega}\right\rangle .
\end{aligned}
$$

Hence we have shown that the scalar Ward identities hold for $n$ factors if one of the factors is a free field.

Step 3): We now show that one can remove the anomaly by a suitable redefinition of the time ordered products. In order to do this, we first show that it is possible to write the $\mathbb{C}$-number distribution $D$ as the total divergence

$$
D\left(y, x_{1}, \ldots, x_{n}\right)=\nabla_{a}^{y} D^{a}\left(y, x_{1}, \ldots, x_{n}\right),
$$


of some vector-valued distribution $D^{a}$ which is supported on the total diagonal of $M^{n+1}$ having microlocal scaling degree $\mu \operatorname{sd}(D)-1$ on the total diagonal and which is invariant under the flow $\psi^{t}$. It is clear that the redefined time ordered products $\mathscr{T}\left(j^{a} A_{1} \ldots A_{n}\right)-$ $D^{a} \cdot \mathbb{1}$ then satisfy the Ward identities and all the other requirements, thus concluding the proof.

Let us call a symbol $b$ "invariant" if there holds $b\left(\psi^{t}(y), \underline{k}\right)=b\left(y, \psi^{t *} \underline{k}\right)$ for all $t \in \mathbb{R}$. In addition to scalar valued symbols, we also want to consider tensor-valued symbols $b^{a_{1} \ldots a_{s}}$. These are defined in the same way as the ordinary symbols above, but with the difference that $b^{a_{1} \ldots a_{s}}(y, \underline{k})$ is now a tensor in the tangent space at $y$. The rank and the principal part of such symbols are defined by analogy to the scalar case. An invariant, tensor-valued symbol is one for which $b^{a_{1} \ldots a_{s}}\left(\psi^{t}(y), \underline{k}\right)=\psi_{*}^{t} b^{a_{1} \ldots a_{s}}\left(y, \psi^{t *} \underline{k}\right)$ for all $t$. Any contraction or tensor product with $\mathbf{g}_{a b}, \mathbf{g}^{a b}, \xi_{a}, k_{a}$ or covariant derivative with respect to $y$ of an invariant, tensor-valued symbol gives again a symbol of that kind. Any distribution $B \in \mathscr{D}^{\prime}\left(M^{n+1}\right)$ which is supported on the total diagonal with microlocal scaling degree $\mu \operatorname{sd}(B)<\infty$ (w.r.t. the total diagonal) and which is addition invariant under the flow $\psi^{t}$ (such as for example $D$ ) arises in the form (12) from an invariant symbol $b$ of degree $\mu \mathrm{sd}(B)-4 n$, and vice-versa. Analogous statements hold true for tensor-valued symbols.

Let $d$ be the invariant symbol corresponding to $D$. Let us assume that there exist vector-valued, invariant symbols $d^{a}$ with the property that

$$
\nabla_{a}^{y} d^{a}(y, \underline{k})+d^{a}(y, \underline{k}) \sum_{j=1}^{n} i k_{j a}=d(y, \underline{k})
$$

for all $(y, \underline{k}) \in T_{y}^{*} M \times \cdots \times T_{y}^{*} M$, and let $D^{a}$ be the corresponding vector-valued, invariant distribution on $M^{n+1}$. Then $D^{a}$ satisfies Eq. (24). It thus remains to construct a vector valued, invariant symbol $d^{a}$ with the property Eq. (25). In order to do that, we start with a lemma.

Lemma 4.1. The distribution $D$ (the anomaly) has the property $D(1 \otimes f)=0$ for all $f \in \mathscr{D}\left(M^{n}\right)$, in other words

$$
\int_{M} D(y, \underline{x}) \mu_{\mathbf{g}}(y)=0 .
$$

Proof. Let us chose a bounded region $\mathcal{O}$ containing the points $x_{1}, \ldots, x_{n}$ and a function $F$ which is equal to one on a neighbourhood of $\mathcal{O}$ and which has the property that $\nabla_{a} F=n_{a} f_{1}-n_{a} f_{2}$ where $f_{1}$ and $f_{2}$ and $n^{a}$ are as under "Technical Data" in the previous section (with $\widehat{\mathcal{O}}$ taken to be $M$ ) and where in addition $\operatorname{supp}\left(f_{1}\right) \cap J^{+}(\mathcal{O})=\emptyset$ and $\operatorname{supp}\left(f_{2}\right) \cap$ 
$J^{-}(\mathcal{O})=\emptyset$. Then by causal factorization,

$$
\begin{aligned}
\int D(y, \underline{x}) F(y) \mu_{\mathbf{g}}(y) & =j^{a}\left(f_{1} n_{a}\right) \mathscr{T}\left(A_{1}\left(x_{1}\right) \ldots A_{n}\left(x_{n}\right)\right)-\mathscr{T}\left(A_{1}\left(x_{1}\right) \ldots A_{n}\left(x_{n}\right)\right) j^{a}\left(f_{2} n_{a}\right) \\
& -i \sum_{j=1}^{n} \xi^{a} \nabla_{a}^{x_{j}} \mathscr{T}\left(A_{1}\left(x_{1}\right) \ldots A_{n}\left(x_{n}\right)\right) \\
& =\left[j^{a}\left(f_{1} n_{a}\right), \mathscr{T}\left(A_{1}\left(x_{1}\right) \ldots A_{n}\left(x_{n}\right)\right)\right]-\mathscr{T}\left(A_{1}\left(x_{1}\right) \ldots A_{n}\left(x_{n}\right)\right) j^{a}\left(\nabla_{a} F\right) \\
& -i \sum_{j=1}^{n} \xi^{a} \nabla_{a}^{x_{j}} \mathscr{T}\left(A_{1}\left(x_{1}\right) \ldots A_{n}\left(x_{n}\right)\right) .
\end{aligned}
$$

The second to last term vanishes by current conservation. Now by Lem. 3.1, we have $\left[j^{a}\left(f_{1} n_{a}\right), \Phi(x)\right]=[H, \Phi(x)]$ for all $x \in \mathcal{O}$. Together with the Wick expansion requirement on the time ordered products, Eq. (91), one can conclude from this that

$$
\left[j^{a}\left(f_{1} n_{a}\right), \mathscr{T}\left(A_{1}\left(x_{1}\right) \ldots A_{n}\left(x_{n}\right)\right)\right]=\left[H, \mathscr{T}\left(A_{1}\left(x_{1}\right) \ldots A_{n}\left(x_{n}\right)\right)\right]
$$

where $H$ is the generator of the group $U(t)$. Hence it remains to show that

$$
\left[H, \mathscr{T}\left(A_{1}\left(x_{1}\right) \ldots A_{n}\left(x_{n}\right)\right)\right]-i \sum_{j=1}^{n} \xi^{a} \nabla_{a}^{x_{j}} \mathscr{T}\left(A_{1}\left(x_{1}\right) \ldots A_{n}\left(x_{n}\right)\right)=0
$$

But this equation is just the infinitesimal version of the covariance requirement, so the lemma is proven.

The lemma says that

$$
\int_{M}[d(y,-i \underline{\nabla}) f](y, \ldots, y) \mu_{\mathbf{g}}(y)=0 \quad \text { for all } f \in \mathscr{D}\left(M^{n}\right) .
$$

We are now going to show that this equation implies the existence of vector-valued, invariant symbols $d^{a}$ satisfying Eq. (25). We do this by an induction in the degree $\rho$ of $d$. If $\rho=0$, then it is easy to see that Eq. (27) already implies $d=0$, so in that case Eq. (25) can trivially be satisfied by choosing $d^{a}=0$. Let us now assume that Eq. (27) had been shown to imply the existence of vector-valued, invariant symbols $d^{a}$ satisfying Eq. (25), whenever the degree of $d$ is less or equal to $\rho-1$. We need to show that we can construct symbols $d^{a}$ with the desired properties also if the degree of $d$ is $\rho$.

To do this, we want to exploit Eq. 27] by testing it with compactly supported functions $f$ of the form $\chi \otimes \eta$, where $\chi=\chi\left(x_{1}\right)$ and $\eta=\eta\left(x_{2}, \ldots, x_{n}\right)$ are testfunctions in $\mathscr{D}(M)$ and $\mathscr{D}\left(M^{n-1}\right)$ respectively. Let us expand $d$ in terms of $k_{1}$,

$$
d=d^{(0) a_{1} \ldots a_{\rho}} k_{1 a_{1}} \ldots k_{1 a_{\rho}}+d^{(1) a_{1} \ldots a_{\rho-1}} k_{1 a_{1}} \ldots k_{1 a_{\rho-1}}+\cdots+d^{(\rho)}
$$


where $d^{(m) a_{1} \ldots a_{\rho-m}}=d^{(m) a_{1} \ldots a_{\rho-m}}\left(y, k_{2}, \ldots, k_{n}\right)$ are tensor-valued, invariant symbols of degree $m$, depending on the indicated arguments. From Eq. (27) with $f=\chi \otimes \eta$ we conclude that

$$
\begin{aligned}
0 & =\sum_{m=0}^{\rho}(-i)^{\rho-m} \int_{M} \nabla_{a_{1}} \ldots \nabla_{a_{\rho-m}} \chi(y)\left[d^{(m) a_{1} \ldots a_{\rho-m}}\left(y,-i \nabla^{x_{2}}, \ldots,-i \nabla^{x_{n}}\right) \eta\right](y, \ldots, y) \mu_{\mathbf{g}}(y) \\
& =\sum_{m=0}^{\rho} i^{\rho-m} \int_{M} \chi(y) \nabla_{a_{1}}^{y} \ldots \nabla_{a_{\rho-m}}^{y}\left[d^{(m) a_{1} \ldots a_{\rho-m}}\left(y,-i \nabla^{x_{2}}, \ldots,-i \nabla^{x_{n}}\right) \eta\right](y, \ldots, y) \mu_{\mathbf{g}}(y) \\
& =: \int_{M} \chi(y)\left[p\left(y, i \nabla^{x_{2}}, \ldots, i \nabla^{x_{n}}\right) \eta\right](y, \ldots, y) \mu_{\mathbf{g}}(y),
\end{aligned}
$$

where the symbol $p$ is defined by the last equation. This implies that

$$
\left[p\left(y,-i \nabla^{x_{2}}, \ldots,-i \nabla^{x_{n}}\right) \eta\right](y, \ldots, y)=0 \text { for all } y \in M \text { and } \eta \in \mathscr{D}\left(M^{n-1}\right) .
$$

It follows from this that the symbol $p$ vanishes (and hence also its principal symbol), in other words

$$
\begin{aligned}
& 0=\sigma_{p}\left(y, k_{2}, \ldots, k_{n}\right)= \\
& \sum_{m=0}^{\rho}\left[\sigma_{d(m)}\left(y, k_{2}, \ldots, k_{n}\right)^{a_{1} \ldots a_{\rho-m}} \sum_{j_{1}, \ldots, j_{\rho-m} \neq 1}(-1)^{\rho-m} k_{j_{1} a_{1}} \ldots k_{j_{\rho-m} a_{\rho-m}}\right] .
\end{aligned}
$$

The point is now that the right side of this equation is actually

$$
=\sigma_{d}\left(y, k_{1}=-k_{2}-\cdots-k_{n}, k_{2}, \ldots, k_{n}\right),
$$

which shows that

$$
\sigma_{d}(y, \underline{k})=b^{a}(y, \underline{k}) \sum_{j=1}^{n} k_{j a}
$$

for some vector-valued, invariant symbol $b^{a}$ of degree less or equal to $\rho-1$. Let us now set

$$
r(y, \underline{k}):=d(y, \underline{k})-\nabla_{a}^{y} b^{a}(y, \underline{k})-b^{a}(y, \underline{k}) \sum_{j=1}^{n} i k_{j a} .
$$

$r$ is by definition an invariant, vector-valued symbol of degree $\leq \rho-1$. By construction, $r$ satisfies satisfies Eq. (27). Thus we can apply the induction hypothesis and conclude that there are invariant, vector-valued symbols $r^{a}$ of degree $\leq \rho-2$ such that

$$
\nabla_{a}^{y} r^{a}(y, \underline{k})+r^{a}(y, \underline{k}) \sum_{j=1}^{n} i k_{j a}=r(y, \underline{k}) .
$$

From this one immediately concludes that the invariant, vector-valued symbol $d^{a}=r^{a}+b^{a}$ satisfies Eq. (25), thus concluding the proof. 


\section{References}

[1] N. N. Boguliubov and D. V. Shirikov: "Introduction to the theory of Quantized Fields," Wiley, New York 1959

[2] R. Brunetti, K. Fredenhagen and M. Köhler: "The microlocal spectrum condition and Wick polynomials on curved spacetimes," Commun. Math. Phys. 180, 633-652 (1996)

[3] R. Brunetti and K. Fredenhagen: "Microlocal Analysis and Interacting Quantum Field Theories: Renormalization on physical backgrounds," Commun. Math. Phys. 208, 623-661 (2000)

[4] T. S. Bunch: "BPHZ renormalization of $\lambda \Phi^{4}$ field theory in curved space-times," Ann. of Phys. 131, 118 (1981)

[5] T. S. Bunch, P. Pannangaden and L. Parker: "On renormalization of $\lambda \Phi^{4}$ in curved space-time I," J. Phys. A: Math. Gen. 13, 901-918 (1980), "On renormalization of $\lambda \Phi^{4}$ in curved space-time II," J. Phys. A: Math. Gen. 13, 919-932 (1980)

[6] M. Dütsch and K. Fredenhagen: "A local (perturbative) construction of observables in gauge theories: The example of QED," Commun. Math. Phys. 203, 71 (1999) hep-th/9807078

[7] H. Epstein and V. Glaser: "The rôle of locality in perturbation theory," Ann. Inst. H. Poincaré Sec. A XIX, 211-295 (1973)

[8] S. W. Hawking: "Particle creation by black holes," Commun. Math. Phys. 43, 199$220(1975)$

[9] S. Hollands: "Aspects of Quantum Field Theory in Curved Spacetime," PhD. thesis, University of York, York 2000

[10] S. Hollands and R.M. Wald: "Local Wick polynomials and time ordered products of quantum fields in curved spacetime," gr-qc/0103074

[11] L. Hörmander: "The Analysis of Linear Partial Differential Operators III," SpringerVerlag, Berlin 1985

[12] B. S. Kay and R. M. Wald: "Theorems on the uniqueness and thermal properties of stationary, nonsingular, quasifree states on spacetimes with a bifurcate Killing horizon," Phys. Rep. 207, 49 (1991) 
[13] D. Prange: "Energy momentum tensor and operator product expansion in local causal perturbation theory," PhD. thesis, Hamburg University, Hamburg 2000, hep-th/0009124

[14] M. J. Radzikowski: "Micro-Local Approach to the Hadamard condition in QFT on Curved Space-Time," Commun. Math. Phys. 179, 529-553 (1996)

[15] M. Scharf: "Finite Quantum Electrodynamics. The Causal Approach," SpringerVerlag, Berlin 1996

[16] R. Stora: "Lagrangian field theory," Summer School of Theoretical Physics about Particle Physics, Les Houches (1971)

[17] E. C. G. Stückelberg and D. Rivier: "Causalité et structure de la matrice S," Helv. Phys. Acta 23, 215 (1950)

[18] R. M. Wald: "Quantum Field Theory in Curved Spacetime and Black Hole Thermodynamics," The University of Chicago Press, Chicago 1994 